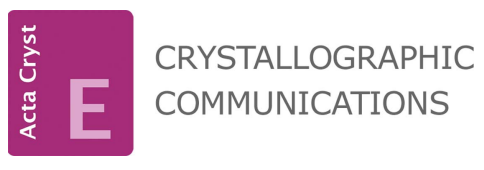

ISSN 2056-9890
Received 2 October 2017

Accepted 6 October 2017

Edited by W. T. A. Harrison, University of Aberdeen, Scotland

Keywords: crystal structure; zinc; cadmium; dithiocarbamate; 4,4'-bipyridine; hydrogen bonding.

CCDC references: 1578328; 1578327

Supporting information: this article has supporting information at journals.iucr.org/e

\section{Crystal structure of $\left(4,4^{\prime}\right.$-bipyridyl- $\left.\kappa N\right)$ bis $[N-(2-$ hydroxyethyl)- $N$-isopropyldithiocarbamato- $\left.\kappa^{2} S, S^{\prime}\right]-$ zinc(II)-4,4'-bipyridyl (2/1) and its isostructural cadmium(II) analogue}

\author{
Yee Seng Tan and Edward R. T. Tiekink*
}

Research Centre for Crystalline Materials, School of Science and Technology, Sunway University, 47500 Bandar Sunway, Selangor Darul Ehsan, Malaysia. *Correspondence e-mail: edwardt@sunway.edu.my

The title structures, $\left[M\left(\mathrm{C}_{6} \mathrm{H}_{12} \mathrm{NOS}_{2}\right)_{2}\left(\mathrm{C}_{10} \mathrm{H}_{8} \mathrm{~N}_{2}\right)\right] \cdot 0.5 \mathrm{C}_{10} \mathrm{H}_{8} \mathrm{~N}_{2}$, for $M=\mathrm{Zn}$, (I), and $\mathrm{Cd}$, (II), feature terminally bound 4,4'-bipyridyl ligands and noncoordinating 4,4'-bipyridyl molecules, with the latter disposed about a centre of inversion. The coordination geometry about the metal atom is defined by two non-symmetrically chelating dithiocarbamate ligands and a pyridyl $\mathrm{N}$ atom. The $\mathrm{NS}_{4}$ donor sets are distorted but, approximate to trigonal bipyramidal in each case. In the crystal, hydroxy-O $-\mathrm{H} \cdots \mathrm{O}$ (hydroxy) and hydroxy-O $-\mathrm{H} \cdots$ $\mathrm{N}$ (pyridyl) hydrogen bonds between the zinc-containing molecules lead to a supramolecular layer parallel to (100). The three-dimensional architecture arises as the layers are linked via methine- $\mathrm{C}-\mathrm{H} \cdots \mathrm{S}$, pyridyl- $\mathrm{C}-\mathrm{H} \cdots$ $\mathrm{O}$ (hydroxy) and $\pi-\pi$ [inter-centroid distance between coordinated pyridyl rings $=3.6246(18) \AA]$ interactions. Channels along the $c$-axis direction are occupied by the non-coordinating 4,4'-bipyridine molecules, which are held in place by $\mathrm{C}-\mathrm{H} \cdots \pi$ (chelate ring) contacts.

\section{Chemical context}

The ditopic ligand 4,4'-bipyridyl is ubiquitous in coordination chemistry, usually providing bridges between metal centres to generate coordination polymers. While bidentate bridging is normally observed in the structural chemistry of zinc(II) $\operatorname{bis}\left(N, N^{\prime}\right.$-dialkyldithiocarbamate)s, these more often than not lead to binuclear species of the general formula $\left[\mathrm{Zn}\left(\mathrm{S}_{2} \mathrm{CN} R R^{\prime}\right)_{2}\right]_{2}\left(4,4^{\prime}\right.$-bipyridyl $)$ as first observed in the archetypal compound $\left[\mathrm{Zn}\left(\mathrm{S}_{2} \mathrm{CNEt}_{2}\right)_{2}\right]_{2}\left(4,4^{\prime}\right.$-bipyridyl) (Zemskova et al., 1994) and in other compounds relevant to the present study, such as $\left\{\mathrm{Zn}\left[\mathrm{S}_{2} \mathrm{CN}(R) \mathrm{CH}_{2} \mathrm{CH}_{2} \mathrm{OH}\right]_{2}\right\}_{2}\left(4,4^{\prime}\right.$ bipyridyl) for $R=\mathrm{Me}$, Et and $\mathrm{CH}_{2} \mathrm{CH}_{2} \mathrm{OH}$ (Benson et al., 2007). The exceptional structure is that of $\mathrm{Zn}\left[\mathrm{S}_{2} \mathrm{CN}(n-\operatorname{Pr})_{2}\right]_{2}$ (4,4'-bipyridyl), which features a relatively rare monodentate coordination mode for the 4,4'-bipyridyl molecule (Klevtsova et al., 2001). The analogous chemistry for cadmium(II) bis( $N, N^{\prime}$-dialkyldithiocarbamate)s is considerably less explored with the only example in the Cambridge Structural Database (Groom et al., 2016) being a linear coordination polymer in the crystal of $\left\{\mathrm{Cd}\left[\mathrm{S}_{2} \mathrm{CN}\left(\mathrm{CH}_{2} \mathrm{Ph}\right)_{2}\right]_{2}\left(4,4^{\prime} \text {-bipyridyl) }\right\}_{n}\right.$ (Fan et al., 2007). The difference in chemistry between zinc and cadmium dithiocarbamates can be rationalized in terms of the larger size of cadmium versus zinc but, also in terms of the reduced Lewis acidity of the zinc atom owing to the strong chelation mode of the dithiocarbamate ligand. This is also true for cadmium whereby unusual coordination modes are found for 
Table 1

Selected geometric parameters $\left(\AA,^{\circ}\right)$ for $(\mathrm{I})$.

\begin{tabular}{lclr}
\hline $\mathrm{Zn}-\mathrm{N} 3$ & $2.077(2)$ & $\mathrm{C} 1-\mathrm{S} 1$ & $1.733(3)$ \\
$\mathrm{Zn}-\mathrm{S} 1$ & $2.3540(10)$ & $\mathrm{C} 1-\mathrm{S} 2$ & $1.715(3)$ \\
$\mathrm{Zn}-\mathrm{S} 2$ & $2.5366(9)$ & $\mathrm{C} 7-\mathrm{S} 3$ & $1.735(3)$ \\
$\mathrm{Zn}-\mathrm{S} 3$ & $2.3541(9)$ & $\mathrm{C} 7-\mathrm{S} 4$ & $1.714(3)$ \\
$\mathrm{Zn}-\mathrm{S} 4$ & $2.5904(9)$ & & \\
$\mathrm{S} 1-\mathrm{Zn}-\mathrm{S} 3$ & $124.19(3)$ & $\mathrm{S} 2-\mathrm{Zn}-\mathrm{S} 4$ & $162.87(3)$ \\
\hline
\end{tabular}

related pyridyl-containing molecules that might otherwise be expected to be bridging. This is discussed further below in Database survey. In the present report, the crystal and molecular structures of two compounds, formulated as $\mathrm{Zn}\left[\mathrm{S}_{2} \mathrm{CN}(i\right.$ Pr) $\left.\mathrm{CH}_{2} \mathrm{CH}_{2} \mathrm{OH}\right]_{2}$ (4,4'-bipyridyl) 0.5 (4,4'-bipyridyl) (I) and the cadmium analogue (II), are described, i.e. featuring monodentate and non-coordinating 4,4'-bipyridine molecules.

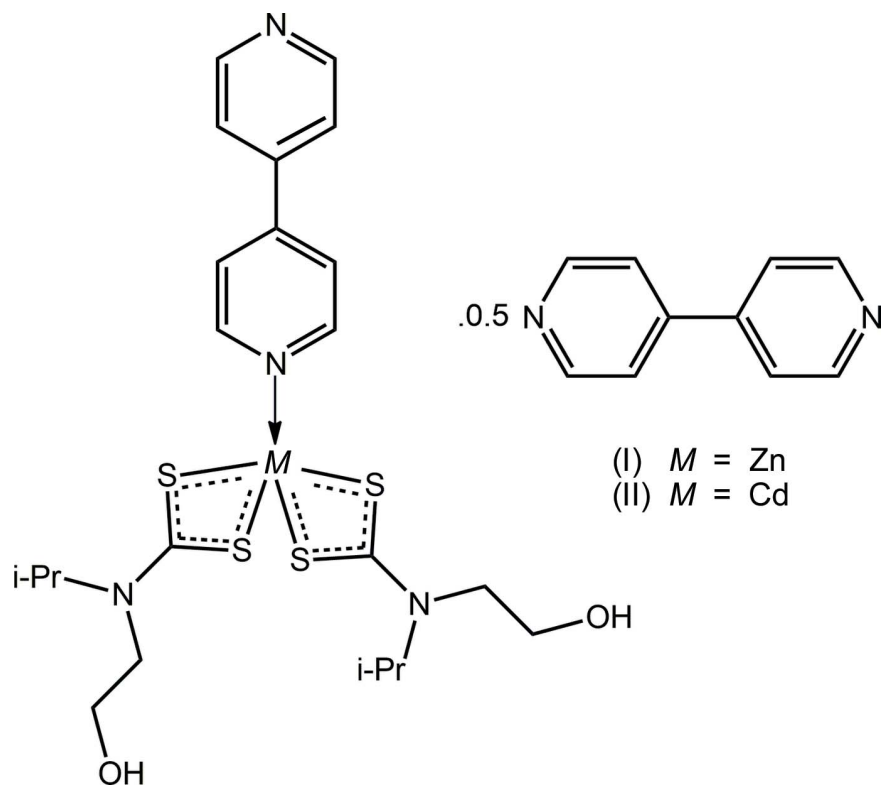

\section{Structural commentary}

The molecular structure of the constituents of (I) are shown in Fig. $1 a$ and selected geometric parameters are collected in Table 1 . The asymmetric unit comprises an entire molecule of $\mathrm{Zn}\left[\mathrm{S}_{2} \mathrm{CN}(i \text {-Pr }) \mathrm{CH}_{2} \mathrm{CH}_{2} \mathrm{OH}\right]_{2}\left(4,4^{\prime}\right.$-bipyridyl $)$ and half a molecule of 4,4'-bipyridine, the latter being disposed about a centre of inversion. The zinc atom is coordinated by two dithiocarbamate ligands that form disparate $\mathrm{Zn}-\mathrm{S}$ bond lengths. This is seen in the values of $\Delta(\mathrm{Zn}-\mathrm{S})=\mathrm{Zn}-\mathrm{S}_{\text {long }}-$ $\mathrm{Zn}-\mathrm{S}_{\text {short }}$, which compute to 0.19 and $0.23 \AA$ for the S1- and S3-dithiocarbamate ligands, respectively. The fifth position in the coordination geometry is occupied by a pyridyl-N atom. Based on the value of $\tau$ (Addison et al., 1984), which equals to 0.0 and 1.0 for ideal square-pyramidal and trigonal-bipyramidal geometries, respectively, it is possible to assign a coordination geometry based on the $\mathrm{NS}_{4}$ donor set. In (I), $\tau=0.64$ indicating a highly distorted coordination geometry but, one approximating a trigonal bipyramid. In this description, the less tightly bound S2 and S4 atoms define the axial
Table 2

Selected geometric parameters $\left(\AA{ }^{\circ},{ }^{\circ}\right)$ for (II).

\begin{tabular}{llll}
\hline $\mathrm{Cd}-\mathrm{N} 3$ & $2.3011(11)$ & $\mathrm{C} 1-\mathrm{S} 1$ & $1.7310(12)$ \\
$\mathrm{Cd}-\mathrm{S} 1$ & $2.5547(3)$ & $\mathrm{C} 1-\mathrm{S} 2$ & $1.7218(12)$ \\
$\mathrm{Cd}-\mathrm{S} 2$ & $2.6500(3)$ & $\mathrm{C} 7-\mathrm{S} 3$ & $1.7328(13)$ \\
$\mathrm{Cd}-\mathrm{S} 3$ & $2.5620(4)$ & $\mathrm{C} 7-\mathrm{S} 4$ & $1.7257(13)$ \\
$\mathrm{Cd}-\mathrm{S} 4$ & $2.6696(4)$ & & \\
& & & \\
$\mathrm{S} 1-\mathrm{Cd}-\mathrm{S} 3$ & $125.725(11)$ & $\mathrm{S} 2-\mathrm{Cd}-\mathrm{S} 4$ & $165.865(11)$ \\
\hline
\end{tabular}

positions, Table 1 . The coordinated $4,4^{\prime}$-bipyridyl molecule is non-planar with the dihedral angle between the two residues being $28.12(14)^{\circ}$.

Crystals of (II) are isostructural to those of (I), Fig. $1 b$ and Table 2. Some differences in molecular geometry are apparent, most notably in the degree of symmetry in the $\mathrm{Cd}-$

(a)

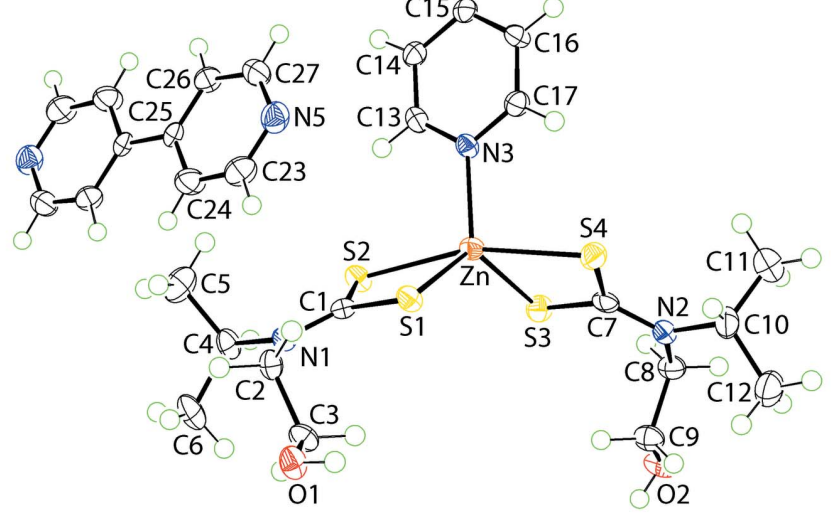

(b)

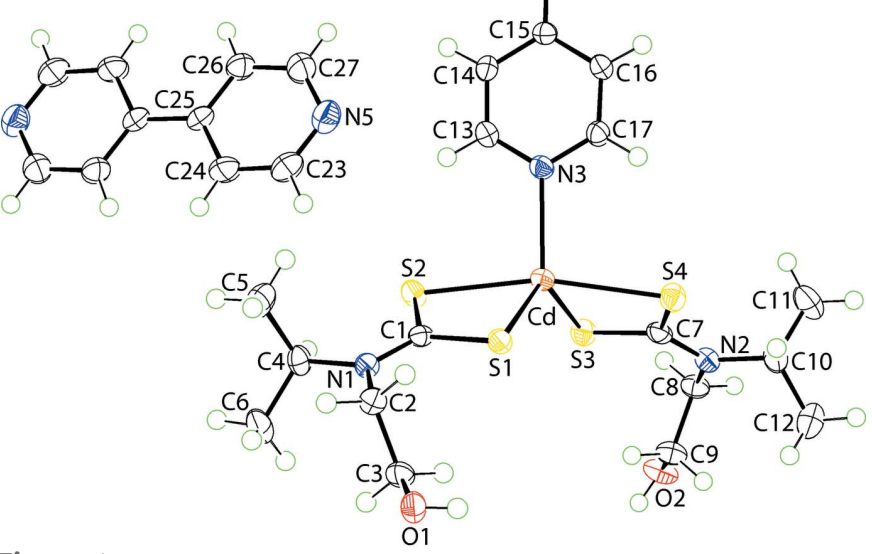

Figure 1

The molecular structures of the constituents of (a) (I) and (b) (II) showing the atom-labelling scheme and displacement ellipsoids at the $70 \%$ probability level. For each of (I) and (II), the 4,4'-bipyridine molecule has been expanded to show the entire molecule; unlabelled atoms are related by the symmetry operation $-x, 2-y,-z$. 
Table 3

Hydrogen-bond geometry $\left(\AA,^{\circ}\right)$ for (II).

$C g 1$ is the centroid of the $\mathrm{Zn} / \mathrm{S} 3 / \mathrm{S} 4 / \mathrm{C} 7$ chelate ring.

\begin{tabular}{lllll}
\hline$D-\mathrm{H} \cdots A$ & $D-\mathrm{H}$ & $\mathrm{H} \cdots A$ & $D \cdots A$ & $D-\mathrm{H} \cdots A$ \\
\hline $\mathrm{O} 1-\mathrm{H} 1 O \cdots \mathrm{N} 4^{\mathrm{i}}$ & $0.83(2)$ & $1.88(2)$ & $2.7085(15)$ & $176(1)$ \\
$\mathrm{O} 2-\mathrm{H} 2 O \cdots{ }^{\mathrm{ii}}$ & $0.83(1)$ & $1.89(1)$ & $2.7162(14)$ & $173(2)$ \\
$\mathrm{C} 4-\mathrm{H} 4 \cdots \mathrm{S}^{\mathrm{iii}}$ & 1.00 & 2.68 & $3.5395(14)$ & 144 \\
$\mathrm{C} 22-\mathrm{H} 22 \cdots \mathrm{O} 2^{\mathrm{iv}}$ & 0.95 & 2.40 & $3.3473(17)$ & 174 \\
$\mathrm{C} 26-\mathrm{H} 26 \cdots C g 1^{\mathrm{v}}$ & 0.95 & 3.00 & $3.776(3)$ & 140 \\
\hline
\end{tabular}

Symmetry codes: (i) $-x, y-1,-z+\frac{1}{2}$; (ii) $x+\frac{1}{2}, y+\frac{1}{2}, z$; (iii) $x+\frac{1}{2}, y+\frac{3}{2}, z$; (iv) $x,-y, z-\frac{1}{2}$; (v) $x, y+1, z$.

Table 4

Hydrogen-bond geometry $\left(\AA,^{\circ}\right)$ for (I).

$C g 1$ is the centroid of the $\mathrm{Cd} / \mathrm{S} 3 / \mathrm{S} 4 / \mathrm{C} 7$ chelate ring.

\begin{tabular}{lllll}
\hline$D-\mathrm{H} \cdots A$ & $D-\mathrm{H}$ & $\mathrm{H} \cdots A$ & $D \cdots A$ & $D-\mathrm{H} \cdots A$ \\
\hline $\mathrm{O} 1-\mathrm{H} 1 O \cdots \mathrm{N} 4^{\mathrm{i}}$ & $0.85(2)$ & $1.85(2)$ & $2.697(3)$ & $179(3)$ \\
$\mathrm{O} 2-\mathrm{H} 2 O \cdots 1^{\mathrm{ii}}$ & $0.84(2)$ & $1.88(2)$ & $2.718(3)$ & $176(4)$ \\
$\mathrm{C} 4-\mathrm{H} 4 \cdots \mathrm{S}^{\mathrm{iii}}$ & 1.00 & 2.67 & $3.515(3)$ & 142 \\
$\mathrm{C} 22-\mathrm{H} 22 \cdots 2^{\mathrm{iv}}$ & 0.95 & 2.36 & $3.300(3)$ & 170 \\
$\mathrm{C} 26-\mathrm{H} 26 \cdots C g 1^{\mathrm{v}}$ & 0.95 & 3.04 & $3.7943(15)$ & 138 \\
\hline
\end{tabular}

Symmetry codes: (i) $-x, y-1,-z+\frac{1}{2}$; (ii) $x+\frac{1}{2}, y+\frac{1}{2}, z$; (iii) $x+\frac{1}{2}, y+\frac{3}{2}, z$; (iv) $x,-y, z-\frac{1}{2}$; (v) $x, y+1, z$.

$\mathrm{S}$ bond lengths, i.e. $\Delta(\mathrm{Cd}-\mathrm{S})=0.09$ and $0.11 \AA$ for the $\mathrm{S} 1-$ and S3-dithiocarbamate ligands, respectively. This is reflected in the narrower ranges in the $\mathrm{C}-\mathrm{S}$ bond lengths in (II) $c f$. (I), Tables 1 and 2 . The value of $\tau=0.67$ suggests a coordination geometry marginally closer to trigonal bipyramidal in (II) than for (I). The dihedral angle between the two rings comprising the coordinated 4,4'-bipyridyl molecule is $28.86(7)^{\circ}$.

\section{Supramolecular features}

The molecular packing of (I) comprises conventional hydrogen bonding as well as a number of weaker, non-covalent interactions, Table 3. The presence of hydroxy-O$\mathrm{H}$. . O (hydroxy) hydrogen bonds leads to the formation of a centrosymmetric, 28-membered $\left\{\cdots \mathrm{HOC}_{2} \mathrm{NCSZnSCNC}_{2} \mathrm{O}\right\}_{2}$ synthon. This ring contains two additional hydroxy- $\mathrm{O}-\mathrm{H} \mathrm{H}$ atoms and these form hydroxy- $\mathrm{O}-\mathrm{H} \cdots \mathrm{N}$ (pyridyl) hydrogen bonds with the non-coordinating end of the monodentate $4,4^{\prime}$-bipyridyl molecules. This network of hydrogen bonds leads to the formation of a two-dimensional array lying parallel to (100), Fig. 2a. These layers are connected into double-layers via methine-C $-\mathrm{H} \cdots \mathrm{S}$ and $\pi-\pi$ interactions involving the coordinated pyridyl ring [inter-centroid distance between the $(\mathrm{N} 3 / \mathrm{C} 13-\mathrm{C} 17)$ and $(\mathrm{N} 3 / \mathrm{C} 13-\mathrm{C} 17)^{\mathrm{i}}$ rings = $3.6246(18) \AA$ and angle of inclination $=0.46(13)^{\circ}$ for symmetry code (i): $\left.-x, y, \frac{1}{2}-z\right]$. The double-layers are connected into a three-dimensional architecture via 4,4'-bipyridyl-C- $\mathrm{H} \cdots \mathrm{O}$ (hydroxy) interactions, involving an $\mathrm{H}$ atom from the non-coordinating ring of the coordinated 4,4'-bipyridyl molecule. This architecture defines channels parallel to the $c$ axis in which residue the non-coordinating $4,4^{\prime}$-bipyridine molecules. The closest interaction between the host and guests are of the type pyridine-C $-\mathrm{H} \cdots \pi(\mathrm{Zn} / \mathrm{S} 3 / \mathrm{S} 4 /$ $\mathrm{C} 7$ ), i.e. $\mathrm{C}-\mathrm{H} \cdots \pi$ (chelate ring), a supramolecular synthon gaining prominence in the structural chemistry of metalcontaining species (Tiekink, 2017), especially for dithiocarbamates (Tiekink \& Zukerman-Schpector, 2011) owing to

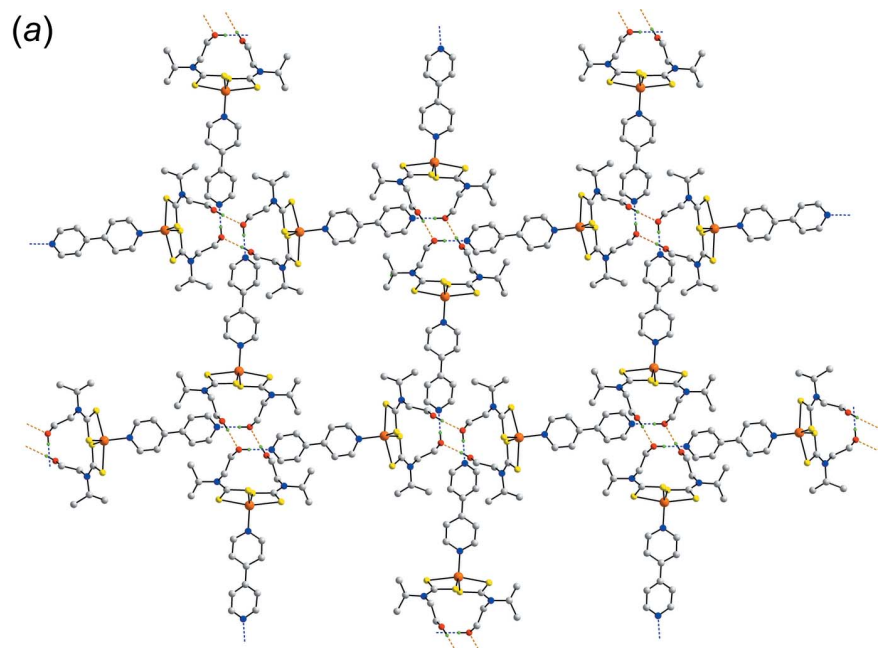

(b)

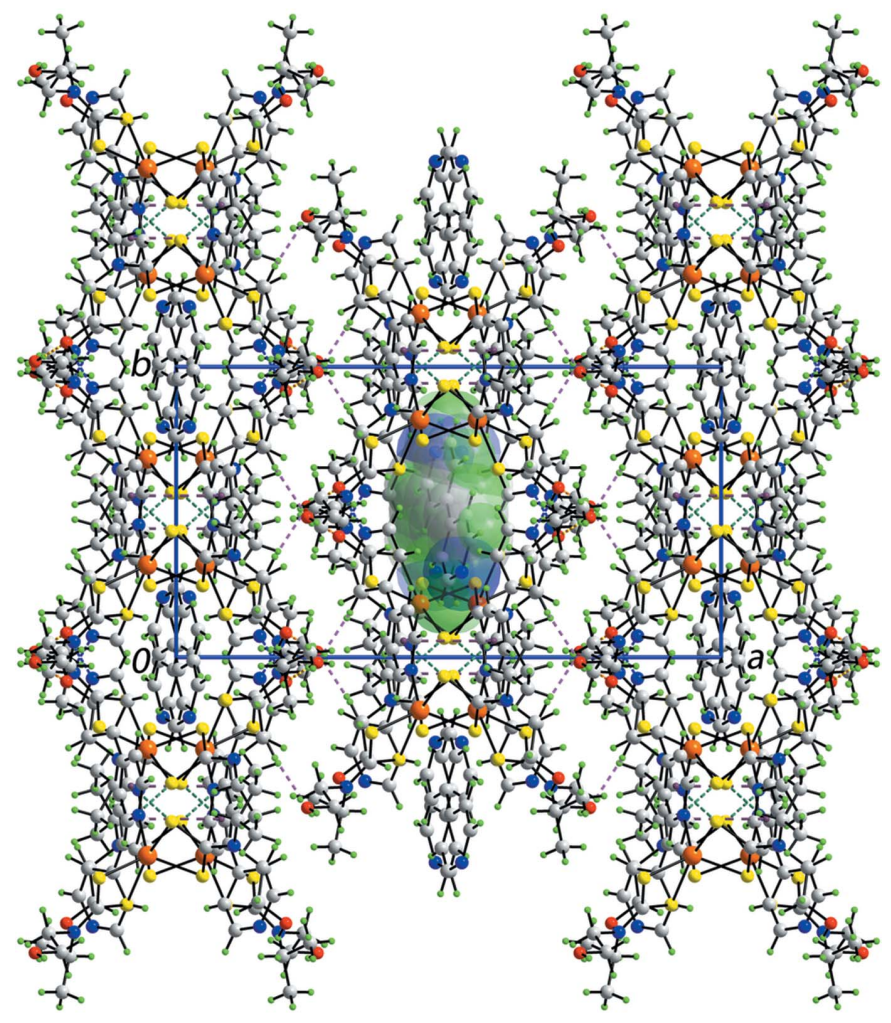

Figure 2

Molecular packing in (I): (a) view of two-dimensional supramolecular array sustained by hydroxy-O $-\mathrm{H} \cdots \mathrm{O}$ (hydroxy) and hydroxy-O$\mathrm{H} \cdots \mathrm{N}$ (pyridyl) hydrogen bonding with all but the acidic $\mathrm{H}$ atoms removed and $(b)$ a view of the unit-cell contents in projection down the $c$ axis, with the non-coordinating 4,4'-bipyridine molecules in one channel highlighted in space-filling mode. The $\mathrm{O}-\mathrm{H} \cdots \mathrm{O}, \mathrm{O}-\mathrm{H} \cdots \mathrm{N}, \mathrm{C}-\mathrm{H} \cdots \mathrm{O}$, $\mathrm{C}-\mathrm{H} \cdots \mathrm{S}$ and $\pi-\pi$ interactions are shown as orange, blue, pink, sea-blue and purple dashed lines, respectively. 
Table 5

Experimental details.

(I)

Crystal data

Chemical formula

$M_{\mathrm{r}}$

Crystal system, space group

Temperature (K)

$a, b, c(\AA)$

$\beta\left({ }^{\circ}\right)$

$V\left(\AA^{3}\right)$

$Z$

Radiation type

$\mu\left(\mathrm{mm}^{-1}\right)$

Crystal size (mm)

Data collection

Diffractometer

Absorption correction

$T_{\min }, T_{\max }$

No. of measured, independent and

observed $[I>2 \sigma(I)]$ reflections

$R_{\text {int }}$

$(\sin \theta / \lambda)_{\max }\left(\AA^{-1}\right)$

Refinemen

$R\left[F^{2}>2 \sigma\left(F^{2}\right)\right], w R\left(F^{2}\right), S$

No. of reflections

No. of parameters

No. of restraints

$\mathrm{H}$-atom treatment

$\Delta \rho_{\max }, \Delta \rho_{\min }\left(\mathrm{e} \AA^{-3}\right)$

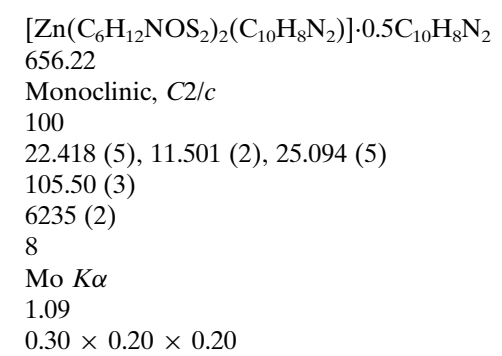

(II)

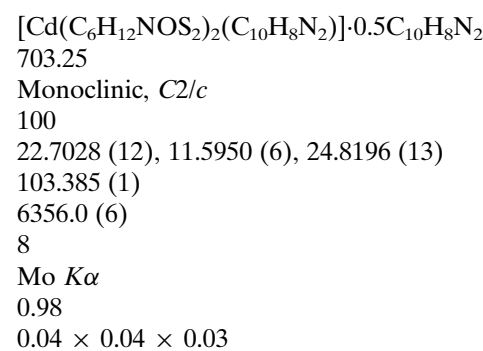

Bruker SMART APEX CCD

Multi-scan (SADABS; Sheldrick, 1996)

$0.968,0.979$

31204, 7738, 5204

0.082

0.667

Bruker SMART APEX CCD

Multi-scan (SADABS; Sheldrick, 1996)

$0.962,0.971$

$41284,7847,7204$

0.023

0.667

$0.041,0.096,1.00$
7738
358
2
$\mathrm{H}$ atoms treated by a mixture of independent
$\quad$ and constrained refinement
$0.48,-0.46$

$0.019,0.049,0.99$

7847

358

2

$\mathrm{H}$ atoms treated by a mixture of independent and constrained refinement

$0.46,-0.24$

Computer programs: APEX2 and SAINT (Bruker, 2008), SHELXS97 (Sheldrick, 2008), SHELXL2014 (Sheldrick, 2015), ORTEP-3 for Windows (Farrugia, 2012), DIAMOND (Brandenburg, 2006) and publCIF (Westrip, 2010).

the ability of the dithiocarbamate ligand to form strong chelating interactions (see above).

The molecular packing for isostructural (II) follows that just described for (I), Table 4. However, in this case, the putative pyridyl-C $-\mathrm{H} \cdots \pi(\mathrm{Cd} / \mathrm{S} 3 / \mathrm{S} 4 / \mathrm{C} 7)$ interaction is just beyond the sum of the van der Waals radii for this type of contact (Spek, 2009).

\section{Database survey}

As mentioned in the Chemical context, ditopic ligands such as 4,4'-bipyridyl are normally observed providing bridges between metal centres. Thus, the structures of (I) and (II) are doubly curious as not only is the $4,4^{\prime}$-bipyridyl ligand coordinating in a monodentate fashion, there is a non-coordinating 4,4'-bipyridine molecule in the crystal. Recent reports confirm these interesting observations with related bipyridyl-type molecules of both the zinc(II) and, especially, cadmium(II) dithiocarbamates. Thus, just as for the 4,4'-bipyridyl structures mentioned in the Chemical context, i.e. $\left[\mathrm{Zn}\left(\mathrm{S}_{2} \mathrm{CNEt}_{2}\right)_{2}\right]_{2}\left(4,4^{\prime}-\right.$ bipyridyl) (Zemskova et al., 1994) and $\mathrm{Zn}\left[\mathrm{S}_{2} \mathrm{CN}(n-\mathrm{Pr})_{2}\right]_{2}\left(4,4^{\prime}\right.$ bipyridyl) (Klevtsova et al., 2001), with the anticipated bidentate, bridging and non-anticipated terminal coordination, respectively, similar chemistry occurs for the ditopic ligand with an ethylene space, i.e. trans-bis(4-pyridyl)ethylene (bpe) where structures of both bridging, i.e. $\left[\mathrm{Zn}\left(\mathrm{S}_{2} \mathrm{CNEt}_{2}\right)_{2}\right]_{2}$ (bpe) (Arman et al., 2009), and terminal, i.e.
$\mathrm{Zn}\left[\mathrm{S}_{2} \mathrm{CN}(n-\mathrm{Pr})_{2}\right]_{2}$ (bpe) (Lai \& Tiekink, 2003), coordination modes are known. Very recently, terminal coordination was found for 4-pyridinealdazine in the structure of $\mathrm{Zn}\left[\mathrm{S}_{2} \mathrm{CN}(\mathrm{Me}) \mathrm{CH}_{2} \mathrm{CH}_{2} \mathrm{OH}_{2}\right]_{2}$ (4-pyridinealdazine) (Broker et al., 2017). In the realm of cadmium dithiocarbamates, the potentially bridging ligand just mentioned occurs in the structure of $\mathrm{Cd}\left[\mathrm{S}_{2} \mathrm{CN}(n-\mathrm{Pr}) \mathrm{CH}_{2} \mathrm{CH}_{2} \mathrm{OH}_{2}\right]_{2}$ (4-pyridinealdazine $)_{2}$ with both being terminally bound (Broker \& Tiekink, 2011). The ditopic ligand bpe was mentioned above. In the case of cadmium dithiocarbamates, a bidentate, bridging mode is seen in the crystal of $\left[\mathrm{Cd}\left(\mathrm{S}_{2} \mathrm{CNEt}_{2}\right)_{2}\right.$ (bpe $\left.)\right]_{n}$ (Chai et al., 2003). However, in another example both bridging and terminal modes, in a 1:2 ratio, are seen in the structure of $\mathrm{Cd}\left[\mathrm{S}_{2} \mathrm{CN}(i \text {-Pr }) \mathrm{CH}_{2} \mathrm{CH}_{2} \mathrm{OH}_{2}\right]_{2}$ (bpe) $)_{3}$ (Jotani et al., 2016). The occurrence of unusual coordination modes for these bipyridyltype ligands indicate additional factors are coming into play, often a competition between hydrogen bonding and $M \leftarrow \mathrm{N}$ donor interactions but, not always as seen in the structure of $\mathrm{Zn}\left[\mathrm{S}_{2} \mathrm{CN}(n-\mathrm{Pr})_{2}\right]_{2}\left(4,4^{\prime}\right.$-bipyridyl) (Klevtsova et al., 2001).

\section{Synthesis and crystallization}

All chemicals and solvents were used as purchased without purification. The melting point was determined using an Krüss KSP1N melting point meter. The IR spectra were obtained by the attenuated total reflectance (ATR) technique on a Perkin Elmer RX1 FTIR spectrophotometer from 4000 to $400 \mathrm{~cm}^{-1}$. 
${ }^{1} \mathrm{H}$ and ${ }^{13} \mathrm{C}$ NMR spectra were recorded at room temperature in DMSO- $d_{6}$ solution on a Bruker Avance $400 \mathrm{MHz}$ NMR spectrometer.

Synthesis of (I): 4,4'-bipyridine (1.79 mmol, $0.28 \mathrm{~g})$ in ethanol $(25 \mathrm{ml})$ was added dropwise to bis( $N$-2-hydroxyethyl, $N$-isopropyldithiocarbamato)zinc(II) $(1.21 \mathrm{mmol}$, $0.51 \mathrm{~g})$ in ethanol $(25 \mathrm{ml})$. The resulting mixture was stirred for $0.5 \mathrm{~h}$ follow by filtration. After a week of slow evaporation of the filtrate, yellow blocks precipitated (yield: $0.698 \mathrm{~g}, 88 \%$; m.p. $445.6 \mathrm{~K})$. IR $\left(\mathrm{cm}^{-1}\right): 1467(\mathrm{~m})[v(\mathrm{C}-\mathrm{N})], 1175(\mathrm{~m})$ $[v(\mathrm{C}-\mathrm{S})] \mathrm{cm}^{-1.1}{ }^{1} \mathrm{H}$ NMR: $\delta 8.78-7.83(m, 12 \mathrm{H}$, aromatic $\mathrm{H})$, $5.14($ sept, $2 \mathrm{H}, \mathrm{NCH}, 6.63 \mathrm{~Hz}), 4.90(t, 2 \mathrm{H}, \mathrm{OH}, 5.38 \mathrm{~Hz}), 3.78-$ $3.64\left(m, 8 \mathrm{H}, \mathrm{NCH}_{2} \mathrm{CH}_{2} \mathrm{O}\right), 1.18\left(d, 12 \mathrm{H}, \mathrm{CH}_{3}, 6.72 \mathrm{~Hz}\right) .{ }^{13} \mathrm{C}$ NMR: $\delta 204.15\left(\mathrm{CS}_{2}\right), 150.53,144.65,121.58$ (aromatic-C), $58.21\left(\mathrm{CH}_{2} \mathrm{O}\right), 55.53\left(\mathrm{NCH}_{2}\right), 49.80(\mathrm{NCH}), 19.88\left(\mathrm{CH}_{3}\right)$.

Synthesis of (II): 4,4'-bipyridine (1.61 mmol, $0.25 \mathrm{~g}$ ) in ethanol $(25 \mathrm{ml})$ was added dropwise to bis( $N$-2-hydroxyethyl, $N$-isopropyldithiocarbamato)cadmium(II) (1.07 mmol, $0.50 \mathrm{~g})$ in ethanol $(25 \mathrm{ml})$. The resulting mixture was stirred for $0.5 \mathrm{~h}$ follow by filtration. A week of slow evaporation of the filtrate yielded yellow blocks (yield: $0.652 \mathrm{~g}, 87 \%$; m.p. $438.7 \mathrm{~K})$. IR $\left(\mathrm{cm}^{-1}\right): 1467(m)[v(\mathrm{C}-\mathrm{N})], 1174(m)[v(\mathrm{C}-\mathrm{S})]$ $\mathrm{cm}^{-1 .}{ }^{1} \mathrm{H}$ NMR: $\delta 8.79-7.80(\mathrm{~m}, 12 \mathrm{H}$, aromatic $\mathrm{H}), 5.22$ (sept, $2 \mathrm{H}, \mathrm{NCH}, 6.63 \mathrm{~Hz}), 4.84(t, 2 \mathrm{H}, \mathrm{OH}, 5.52 \mathrm{~Hz}), 3.80-3.64(m$, $\left.8 \mathrm{H}, \mathrm{NCH}_{2} \mathrm{CH}_{2} \mathrm{O}\right), 1.17\left(d, 12 \mathrm{H}, \mathrm{CH}_{3}, 6.72 \mathrm{~Hz}\right) \cdot{ }^{13} \mathrm{C}$ NMR: $\delta$ $205.29\left(\mathrm{CS}_{2}\right), 150.57,144.46,121.41$ (aromatic-C), 58.26 $\left(\mathrm{CH}_{2} \mathrm{O}\right), 56.62\left(\mathrm{NCH}_{2}\right), 50.47(\mathrm{NCH}), 19.91\left(\mathrm{CH}_{3}\right)$.

\section{Refinement}

Crystal data, data collection and structure refinement details are summarized in Table 5. For each of (I) and (II), carbonbound $\mathrm{H}$ atoms were placed in calculated positions $(\mathrm{C}-\mathrm{H}=$ $0.95-1.00 \AA$ ) and were included in the refinement in the riding-model approximation, with $U_{\text {iso }}(\mathrm{H})$ set to $1.2-$ $1.5 U_{\text {eq }}(\mathrm{C})$. The $\mathrm{O}$-bound $\mathrm{H}$ atoms were located in differenceFourier maps but were refined with a distance restraint of $\mathrm{O}-$ $\mathrm{H}=0.84 \pm 0.01 \AA$, and with $U_{\text {iso }}(\mathrm{H})$ set to $1.5 U_{\text {eq }}(\mathrm{O})$. For $(\mathrm{I})$, owing to poor agreement, two reflections, i.e. $\left(\begin{array}{lll}0 & 0 & 6\end{array}\right)$ and (27 3 4), were omitted from the final cycles of refinement. For
(II), one reflection, i.e. $(\overline{27} 77)$, was omitted for the same reason.

\section{Acknowledgements}

Sunway University is thanked for support of biological and crystal engineering studies of metal dithiocarbamates.

\section{References}

Addison, A. W., Rao, T. N., Reedijk, J., van Rijn, J. \& Verschoor, G. C. (1984). J. Chem. Soc. Dalton Trans. pp. 1349-1356.

Arman, H. D., Poplaukhin, P. \& Tiekink, E. R. T. (2009). Acta Cryst. E65, m1472-m1473.

Benson, R. E., Ellis, C. A., Lewis, C. E. \& Tiekink, E. R. T. (2007). CrystEngComm, 9, 930-941.

Brandenburg, K. (2006). DIAMOND. Crystal Impact GbR, Bonn, Germany.

Broker, G. A., Jotani, M. M. \& Tiekink, E. R. T. (2017). Acta Cryst. E73, 1458-1464.

Broker, G. A. \& Tiekink, E. R. T. (2011). Acta Cryst. E67, m320m321.

Bruker (2008). APEX2 and SAINT. Bruker AXS Inc., Madison, Wisconsin, USA.

Chai, J., Lai, C. S., Yan, J. \& Tiekink, E. R. T. (2003). Appl. Organomet. Chem. 17, 249-250.

Fan, J., Wei, F.-X., Zhang, W.-G., Yin, X., Lai, C.-S. \& Tiekink, E. R. T. (2007). Acta Chim. Sinica, 65, 2014-2018.

Farrugia, L. J. (2012). J. Appl. Cryst. 45, 849-854.

Groom, C. R., Bruno, I. J., Lightfoot, M. P. \& Ward, S. C. (2016). Acta Cryst. B72, 171-179.

Jotani, M. M., Arman, H. D., Poplaukhin, P. \& Tiekink, E. R. T. (2016). Acta Cryst. E72, 1700-1709.

Klevtsova, R. F., Glinskaya, L. A., Berus, E. I. \& Larionov, S. V. (2001). J. Struct. Chem. 42, 639-647.

Lai, C. S. \& Tiekink, E. R. T. (2003). Appl. Organomet. Chem. 17, 251-252.

Sheldrick, G. M. (1996). SADABS University of Göttingen, Germany.

Sheldrick, G. M. (2008). Acta Cryst. A64, 112-122.

Sheldrick, G. M. (2015). Acta Cryst. C71, 3-8.

Spek, A. L. (2009). Acta Cryst. D65, 148-155.

Tiekink, E. R. T. (2017). Coord. Chem. Rev. 345, 209-228.

Tiekink, E. R. T. \& Zukerman-Schpector, J. (2011). Chem. Commun. 47, 6623-6625.

Westrip, S. P. (2010). J. Appl. Cryst. 43, 920-925.

Zemskova, S. M., Glinskaya, L. A., Durasov, V. B., Klevtsova, R. F. \& Larionov, S. V. (1994). J. Struct. Chem. 34, 794-802. 


\section{supporting information}

Acta Cryst. (2017). E73, 1642-1646 [https://doi.org/10.1107/S2056989017014396]

Crystal structure of $\left(4,4^{\prime}\right.$-bipyridyl- $\left.\kappa \mathrm{N}\right)$ bis[N-(2-hydroxyethyl)-N-isopropyldithiocarbamato- $\left.\kappa^{2} S, S^{\prime}\right]$ zinc(II)-4,4'-bipyridyl (2/1) and its isostructural cadmium(II) analogue

\section{Yee Seng Tan and Edward R. T. Tiekink}

Computing details

For both structures, data collection: APEX2 (Bruker, 2008); cell refinement: SAINT (Bruker, 2008); data reduction: SAINT (Bruker, 2008); program(s) used to solve structure: SHELXS97 (Sheldrick, 2008); program(s) used to refine structure: SHELXL2014 (Sheldrick, 2015); molecular graphics: ORTEP-3 for Windows (Farrugia, 2012) and DIAMOND (Brandenburg, 2006); software used to prepare material for publication: publCIF (Westrip, 2010).

(4,4'-Bipyridyl- $\kappa N)$ bis $\left[N\right.$-(2-hydroxyethyl)- $N$-isopropyldithiocarbamato- $\left.\kappa^{2} S, S^{\prime}\right]$ zinc(II)-4,4'-bipyridyl (2/1) (I)

Crystal data

$\left[\mathrm{Zn}\left(\mathrm{C}_{6} \mathrm{H}_{12} \mathrm{NOS}_{2}\right)_{2}\left(\mathrm{C}_{10} \mathrm{H}_{8} \mathrm{~N}_{2}\right)\right] \cdot 0.5 \mathrm{C}_{10} \mathrm{H}_{8} \mathrm{~N}_{2}$ $M_{r}=656.22$

Monoclinic, $C 2 / c$

$a=22.418(5) \AA$

$b=11.501(2) \AA$

$c=25.094(5) \AA$

$\beta=105.50(3)^{\circ}$

$V=6235(2) \AA^{3}$

$Z=8$

$F(000)=2744$

$D_{\mathrm{x}}=1.398 \mathrm{Mg} \mathrm{m}^{-3}$

Mo $K \alpha$ radiation, $\lambda=0.71073 \AA$

Cell parameters from 3045 reflections

$\theta=2.3-25.2^{\circ}$

$\mu=1.09 \mathrm{~mm}^{-1}$

$T=100 \mathrm{~K}$

Block, yellow

$0.30 \times 0.20 \times 0.20 \mathrm{~mm}$

Data collection

Bruker SMART APEX CCD diffractometer

Radiation source: fine-focus sealed tube Graphite monochromator $\omega$ scans

Absorption correction: multi-scan (SADABS; Sheldrick, 1996)

$T_{\min }=0.968, T_{\max }=0.979$

31204 measured reflections

7738 independent reflections

5204 reflections with $I>2 \sigma(I)$

$R_{\text {int }}=0.082$

$\theta_{\text {max }}=28.3^{\circ}, \theta_{\text {min }}=2.0^{\circ}$

$h=-27 \rightarrow 29$

$k=-15 \rightarrow 15$

$l=-33 \rightarrow 33$

Refinement

Refinement on $F^{2}$

Least-squares matrix: full

$R\left[F^{2}>2 \sigma\left(F^{2}\right)\right]=0.041$

$w R\left(F^{2}\right)=0.096$

$S=1.00$

7738 reflections

358 parameters

2 restraints

Primary atom site location: structure-invariant direct methods

Hydrogen site location: mixed

$\mathrm{H}$ atoms treated by a mixture of independent and constrained refinement

$w=1 /\left[\sigma^{2}\left(F_{\mathrm{o}}^{2}\right)+(0.0351 P)^{2}\right]$ where $P=\left(F_{\mathrm{o}}^{2}+2 F_{\mathrm{c}}^{2}\right) / 3$

$(\Delta / \sigma)_{\max }<0.001$ 
$\Delta \rho_{\max }=0.48$ e $\AA^{-3}$

$\Delta \rho_{\min }=-0.46$ e $\AA^{-3}$

Special details

Geometry. All esds (except the esd in the dihedral angle between two 1.s. planes) are estimated using the full covariance matrix. The cell esds are taken into account individually in the estimation of esds in distances, angles and torsion angles; correlations between esds in cell parameters are only used when they are defined by crystal symmetry. An approximate (isotropic) treatment of cell esds is used for estimating esds involving l.s. planes.

Fractional atomic coordinates and isotropic or equivalent isotropic displacement parameters $\left(\hat{A}^{2}\right)$

\begin{tabular}{|c|c|c|c|c|}
\hline & $x$ & $y$ & $z$ & $U_{\text {iso }} * / U_{\text {eq }}$ \\
\hline $\mathrm{Zn}$ & $0.05280(2)$ & $0.31725(3)$ & $0.16518(2)$ & $0.01632(9)$ \\
\hline S1 & $-0.04838(3)$ & $0.24709(6)$ & $0.12760(3)$ & $0.01626(15)$ \\
\hline S2 & $0.00828(3)$ & $0.43350(6)$ & $0.07767(3)$ & $0.01723(15)$ \\
\hline S3 & $0.14139(3)$ & $0.24004(6)$ & $0.14471(3)$ & $0.01887(16)$ \\
\hline S4 & $0.09136(3)$ & $0.14774(6)$ & $0.23371(3)$ & $0.01927(16)$ \\
\hline $\mathrm{O} 1$ & $-0.20135(9)$ & $0.08393(16)$ & $0.01233(8)$ & $0.0200(4)$ \\
\hline $\mathrm{H} 1 \mathrm{O}$ & $-0.1869(13)$ & $0.034(2)$ & $0.0372(9)$ & $0.030^{*}$ \\
\hline $\mathrm{O} 2$ & $0.25910(9)$ & $-0.01819(18)$ & $0.09286(8)$ & $0.0250(5)$ \\
\hline $\mathrm{H} 2 \mathrm{O}$ & $0.2425(14)$ & $-0.036(3)$ & $0.0599(6)$ & $0.038^{*}$ \\
\hline N1 & $-0.10592(10)$ & $0.35160(18)$ & $0.03253(9)$ & $0.0138(5)$ \\
\hline $\mathrm{N} 2$ & $0.18772(10)$ & $0.05318(19)$ & $0.20497(9)$ & $0.0162(5)$ \\
\hline N3 & $0.06894(10)$ & $0.45571(18)$ & $0.22034(9)$ & $0.0147(5)$ \\
\hline N4 & $0.15344(12)$ & $0.9270(2)$ & $0.40786(10)$ & $0.0240(6)$ \\
\hline N5 & $0.02413(12)$ & $0.7932(2)$ & $0.11006(10)$ & $0.0260(6)$ \\
\hline $\mathrm{C} 1$ & $-0.05450(12)$ & $0.3457(2)$ & $0.07419(11)$ & $0.0140(5)$ \\
\hline $\mathrm{C} 2$ & $-0.15890(12)$ & $0.2760(2)$ & $0.03229(11)$ & $0.0157(6)$ \\
\hline $\mathrm{H} 2 \mathrm{~A}$ & -0.1974 & 0.3119 & 0.0093 & $0.019 *$ \\
\hline $\mathrm{H} 2 \mathrm{~B}$ & -0.1630 & 0.2682 & 0.0704 & $0.019^{*}$ \\
\hline $\mathrm{C} 3$ & $-0.15066(13)$ & $0.1563(2)$ & $0.00952(11)$ & $0.0180(6)$ \\
\hline $\mathrm{H} 3 \mathrm{~A}$ & -0.1488 & 0.1632 & -0.0293 & $0.022 *$ \\
\hline H3B & -0.1114 & 0.1214 & 0.0314 & $0.022 *$ \\
\hline $\mathrm{C} 4$ & $-0.11249(13)$ & $0.4398(2)$ & $-0.01199(11)$ & $0.0183(6)$ \\
\hline $\mathrm{H} 4$ & -0.0699 & 0.4635 & -0.0129 & $0.022 *$ \\
\hline $\mathrm{C} 5$ & $-0.14485(14)$ & $0.5472(2)$ & $0.00182(13)$ & $0.0280(7)$ \\
\hline $\mathrm{H} 5 \mathrm{~A}$ & -0.1490 & 0.6049 & -0.0277 & $0.042 *$ \\
\hline H5B & -0.1860 & 0.5258 & 0.0052 & $0.042^{*}$ \\
\hline $\mathrm{H} 5 \mathrm{C}$ & -0.1204 & 0.5802 & 0.0369 & $0.042 *$ \\
\hline C6 & $-0.14513(14)$ & $0.3914(3)$ & $-0.06898(11)$ & $0.0265(7)$ \\
\hline H6A & -0.1484 & 0.4524 & -0.0969 & $0.040 *$ \\
\hline H6B & -0.1213 & 0.3259 & -0.0776 & $0.040^{*}$ \\
\hline $\mathrm{H} 6 \mathrm{C}$ & -0.1867 & 0.3647 & -0.0691 & $0.040 *$ \\
\hline $\mathrm{C} 7$ & $0.14472(13)$ & $0.1375(2)$ & $0.19613(11)$ & $0.0167(6)$ \\
\hline $\mathrm{C} 8$ & $0.23385(12)$ & $0.0495(2)$ & $0.17274(11)$ & $0.0185(6)$ \\
\hline H8A & 0.2719 & 0.0110 & 0.1950 & $0.022^{*}$ \\
\hline $\mathrm{H} 8 \mathrm{~B}$ & 0.2448 & 0.1300 & 0.1650 & $0.022 *$ \\
\hline C9 & $0.21025(13)$ & $-0.0148(3)$ & $0.11867(11)$ & $0.0208(6)$ \\
\hline H9A & 0.1976 & -0.0947 & 0.1255 & $0.025^{*}$ \\
\hline H9B & 0.1740 & 0.0259 & 0.0947 & $0.025^{*}$ \\
\hline
\end{tabular}




$\begin{array}{lllll}\text { C10 } & 0.19577(13) & -0.0287(2) & 0.25202(11) & 0.0216(6) \\ \text { H10 } & 0.1560 & -0.0303 & 0.2629 & 0.026^{*} \\ \text { C11 } & 0.24603(15) & 0.0160(3) & 0.30141(12) & 0.0342(8) \\ \text { H11A } & 0.2511 & -0.0384 & 0.3324 & 0.051^{*} \\ \text { H11B } & 0.2852 & 0.0227 & 0.2912 & 0.051^{*} \\ \text { H11C } & 0.2341 & 0.0925 & 0.3124 & 0.051^{*} \\ \text { C12 } & 0.20898(15) & -0.1525(2) & 0.23619(13) & 0.0294(7) \\ \text { H12A } & 0.2140 & -0.2037 & 0.2683 & 0.044^{*} \\ \text { H12B } & 0.1744 & -0.1801 & 0.2061 & 0.044^{*} \\ \text { H12C } & 0.2471 & -0.1532 & 0.2241 & 0.044^{*} \\ \text { C13 } & 0.06136(13) & 0.5664(2) & 0.20303(11) & 0.0190(6) \\ \text { H13 } & 0.0458 & 0.5809 & 0.1645 & 0.023^{*} \\ \text { C14 } & 0.07504(12) & 0.6604(2) & 0.23849(11) & 0.0182(6) \\ \text { H14 } & 0.0692 & 0.7373 & 0.2242 & 0.022^{*} \\ \text { C15 } & 0.09742(12) & 0.6424(2) & 0.29521(11) & 0.0158(6) \\ \text { C16 } & 0.10366(12) & 0.5272(2) & 0.31327(11) & 0.0165(6) \\ \text { H16 } & 0.1175 & 0.5102 & 0.3517 & 0.020^{*} \\ \text { C17 } & 0.08975(12) & 0.4380(2) & 0.27525(11) & 0.0169(6) \\ \text { H17 } & 0.0951 & 0.3602 & 0.2884 & 0.020^{*} \\ \text { C18 } & 0.11560(13) & 0.7408(2) & 0.33461(11) & 0.0172(6) \\ \text { C19 } & 0.08885(13) & 0.8507(2) & 0.32338(12) & 0.0208(6) \\ \text { H19 } & 0.0571 & 0.8641 & 0.2903 & 0.025^{*} \\ \text { C20 } & 0.10898(14) & 0.9398(2) & 0.36078(12) & 0.0235(7) \\ \text { H20 } & 0.0902 & 1.0141 & 0.3526 & 0.028^{*} \\ \text { C21 } & 0.17857(14) & 0.8211(2) & 0.41830(12) & 0.0251(7) \\ \text { H21 } & 0.2101 & 0.8102 & 0.4517 & 0.030^{*} \\ \text { C22 } & 0.16164(13) & 0.7269(2) & 0.38367(11) & 0.0192(6) \\ \text { H22 } & 0.1811 & 0.6536 & 0.3932 & 0.023^{*} \\ \text { C23 } & -0.00233(15) & 0.7643(2) & 0.05770(13) & 0.0277(7) \\ \text { H23 } & -0.0148 & 0.6858 & 0.0501 & 0.033^{*} \\ \text { C24 } & -0.01294(14) & 0.8414(2) & 0.01340(12) & 0.0256(7) \\ \text { H24 } & -0.0318 & 0.8151 & -0.0231 & 0.031^{*} \\ \text { C25 } & 0.00438(12) & 0.9574(2) & 0.02306(11) & 0.0152(6) \\ \text { C26 } & 0.03155(13) & 0.9879(2) & 0.07766(11) & 0.0218(6) \\ \text { H26 } & 0.0444 & 1.0658 & 0.0868 & 0.026^{*} \\ \text { C27 } & 0.03987(14) & 0.9051(3) & 0.11860(12) & 0.0260(7) \\ \text { H27 } & 0.0581 & 0.9292 & 0.1556 & 0.031^{*} \\ & & & & \end{array}$

Atomic displacement parameters $\left(\AA^{2}\right)$

\begin{tabular}{lllllll}
\hline & $U^{11}$ & $U^{22}$ & $U^{33}$ & $U^{12}$ & $U^{13}$ & $U^{23}$ \\
\hline Zn & $0.01770(18)$ & $0.01443(16)$ & $0.01542(16)$ & $-0.00168(14)$ & $0.00199(13)$ & $-0.00196(13)$ \\
S1 & $0.0195(4)$ & $0.0144(3)$ & $0.0137(3)$ & $-0.0037(3)$ & $0.0024(3)$ & $0.0018(3)$ \\
S2 & $0.0190(4)$ & $0.0162(3)$ & $0.0149(3)$ & $-0.0062(3)$ & $0.0019(3)$ & $0.0018(3)$ \\
S3 & $0.0212(4)$ & $0.0169(3)$ & $0.0191(4)$ & $-0.0009(3)$ & $0.0062(3)$ & $0.0018(3)$ \\
S4 & $0.0232(4)$ & $0.0183(3)$ & $0.0172(4)$ & $0.0032(3)$ & $0.0071(3)$ & $0.0019(3)$ \\
O1 & $0.0201(11)$ & $0.0164(10)$ & $0.0207(11)$ & $-0.0046(8)$ & $0.0006(9)$ & $0.0059(8)$ \\
O2 & $0.0193(11)$ & $0.0349(12)$ & $0.0216(11)$ & $-0.0052(9)$ & $0.0066(9)$ & $-0.0113(10)$
\end{tabular}




\begin{tabular}{|c|c|c|c|c|c|c|}
\hline N1 & $0.0157(12)$ & $0.0126(11)$ & $0.0126(11)$ & $-0.0023(9)$ & $0.0031(9)$ & $0.0012(8)$ \\
\hline N2 & $0.0159(12)$ & $0.0178(12)$ & $0.0139(11)$ & $0.0007(10)$ & $0.0020(9)$ & $-0.0006(9)$ \\
\hline N3 & $0.0134(12)$ & $0.0159(11)$ & $0.0134(11)$ & $-0.0017(9)$ & $0.0010(9)$ & $-0.0001(9)$ \\
\hline N4 & $0.0313(15)$ & $0.0186(12)$ & $0.0204(13)$ & $0.0010(11)$ & $0.0039(11)$ & $-0.0049(10)$ \\
\hline N5 & $0.0279(15)$ & $0.0205(13)$ & $0.0284(14)$ & 0.0004 (11) & $0.0055(12)$ & $0.0037(11)$ \\
\hline $\mathrm{C} 1$ & $0.0161(14)$ & $0.0120(12)$ & $0.0146(13)$ & $-0.0001(11)$ & $0.0050(11)$ & $-0.0028(10)$ \\
\hline $\mathrm{C} 2$ & 0.0113 (14) & $0.0175(13)$ & $0.0176(14)$ & $-0.0014(11)$ & $0.0025(11)$ & 0.0039 (11) \\
\hline $\mathrm{C} 3$ & $0.0165(15)$ & $0.0202(14)$ & $0.0163(14)$ & $-0.0059(11)$ & $0.0026(11)$ & $-0.0010(11)$ \\
\hline $\mathrm{C} 4$ & $0.0195(15)$ & $0.0186(14)$ & $0.0150(14)$ & $-0.0025(12)$ & 0.0014 (11) & 0.0064 (11) \\
\hline $\mathrm{C} 5$ & $0.0345(19)$ & $0.0170(15)$ & $0.0354(18)$ & $0.0029(13)$ & $0.0142(15)$ & $0.0076(13)$ \\
\hline C6 & $0.0271(18)$ & $0.0318(17)$ & $0.0162(15)$ & $-0.0034(14)$ & $-0.0020(13)$ & $0.0073(13)$ \\
\hline $\mathrm{C} 7$ & $0.0175(15)$ & $0.0154(13)$ & $0.0147(14)$ & $-0.0044(11)$ & $0.0003(11)$ & $-0.0047(10)$ \\
\hline $\mathrm{C} 8$ & $0.0153(15)$ & $0.0213(14)$ & $0.0189(14)$ & $-0.0005(12)$ & $0.0044(12)$ & $-0.0045(11)$ \\
\hline C9 & $0.0166(15)$ & $0.0242(15)$ & $0.0216(15)$ & $-0.0037(12)$ & $0.0047(12)$ & $-0.0049(12)$ \\
\hline $\mathrm{C} 10$ & $0.0215(16)$ & $0.0227(15)$ & $0.0200(15)$ & $0.0053(13)$ & $0.0046(12)$ & $0.0054(12)$ \\
\hline $\mathrm{C} 11$ & $0.039(2)$ & $0.0365(19)$ & $0.0228(17)$ & $0.0076(16)$ & $0.0007(15)$ & $0.0012(14)$ \\
\hline C12 & 0.0344 (19) & $0.0234(16)$ & 0.0299 (18) & $0.0057(14)$ & $0.0076(15)$ & $0.0068(13)$ \\
\hline C13 & $0.0201(15)$ & $0.0198(14)$ & $0.0143(14)$ & $0.0016(12)$ & $-0.0002(12)$ & $0.0007(11)$ \\
\hline $\mathrm{C} 14$ & $0.0198(15)$ & $0.0136(13)$ & $0.0195(14)$ & $0.0014(11)$ & $0.0022(12)$ & $-0.0002(11)$ \\
\hline $\mathrm{C} 15$ & 0.0115 (14) & $0.0179(14)$ & $0.0181(14)$ & $0.0011(11)$ & $0.0042(11)$ & $-0.0021(11)$ \\
\hline $\mathrm{C} 16$ & $0.0193(15)$ & $0.0183(14)$ & $0.0115(13)$ & $-0.0017(12)$ & $0.0035(11)$ & $-0.0010(11)$ \\
\hline C17 & $0.0191(15)$ & $0.0138(13)$ & $0.0179(14)$ & $-0.0003(11)$ & $0.0050(12)$ & $0.0010(11)$ \\
\hline $\mathrm{C} 18$ & $0.0200(16)$ & $0.0154(13)$ & $0.0172(14)$ & $-0.0008(11)$ & $0.0067(12)$ & $-0.0008(11)$ \\
\hline $\mathrm{C} 19$ & $0.0212(16)$ & $0.0180(14)$ & $0.0206(15)$ & $0.0032(12)$ & $0.0012(12)$ & $-0.0015(11)$ \\
\hline $\mathrm{C} 20$ & $0.0289(17)$ & $0.0160(14)$ & $0.0250(16)$ & $0.0019(13)$ & $0.0065(13)$ & $-0.0040(12)$ \\
\hline C21 & $0.0324(18)$ & $0.0223(15)$ & $0.0161(14)$ & $0.0017(14)$ & $-0.0013(13)$ & $-0.0025(12)$ \\
\hline $\mathrm{C} 22$ & $0.0239(16)$ & $0.0163(14)$ & $0.0166(14)$ & $0.0008(12)$ & $0.0042(12)$ & $-0.0023(11)$ \\
\hline $\mathrm{C} 23$ & $0.0342(19)$ & $0.0154(14)$ & $0.0330(18)$ & $-0.0033(13)$ & $0.0077(15)$ & $-0.0007(13)$ \\
\hline $\mathrm{C} 24$ & $0.0342(19)$ & $0.0179(15)$ & $0.0233(16)$ & $-0.0031(13)$ & $0.0051(14)$ & $-0.0013(12)$ \\
\hline $\mathrm{C} 25$ & $0.0120(14)$ & $0.0135(13)$ & $0.0203(14)$ & $0.0026(11)$ & $0.0049(11)$ & $0.0014(11)$ \\
\hline $\mathrm{C} 26$ & $0.0270(17)$ & 0.0109 (13) & $0.0255(16)$ & $-0.0030(12)$ & $0.0035(13)$ & $-0.0013(11)$ \\
\hline $\mathrm{C} 27$ & $0.0295(18)$ & $0.0244(16)$ & $0.0208(16)$ & $-0.0003(14)$ & $0.0009(13)$ & $-0.0022(13)$ \\
\hline
\end{tabular}

Geometric parameters $\left(\AA,{ }^{\circ}\right)$

\begin{tabular}{llll}
\hline $\mathrm{Zn}-\mathrm{N} 3$ & $2.077(2)$ & $\mathrm{C} 8-\mathrm{H} 8 \mathrm{~A}$ & 0.9900 \\
$\mathrm{Zn}-\mathrm{S} 1$ & $2.3540(10)$ & $\mathrm{C} 8-\mathrm{H} 8 \mathrm{~B}$ & 0.9900 \\
$\mathrm{Zn}-\mathrm{S} 2$ & $2.5366(9)$ & $\mathrm{C} 9-\mathrm{H} 9 \mathrm{~A}$ & 0.9900 \\
$\mathrm{Zn}-\mathrm{S} 3$ & $2.3541(9)$ & $\mathrm{C} 9-\mathrm{H} 9 \mathrm{~B}$ & 0.9900 \\
$\mathrm{Zn}-\mathrm{S} 4$ & $2.5904(9)$ & $\mathrm{C} 10-\mathrm{C} 11$ & $1.525(4)$ \\
$\mathrm{C} 1-\mathrm{S} 1$ & $1.733(3)$ & $\mathrm{C} 10-\mathrm{C} 12$ & $1.529(4)$ \\
$\mathrm{C} 1-\mathrm{S} 2$ & $1.715(3)$ & $\mathrm{C} 10-\mathrm{H} 10$ & 1.0000 \\
$\mathrm{C} 7-\mathrm{S} 3$ & $1.735(3)$ & $\mathrm{C} 11-\mathrm{H} 11 \mathrm{~A}$ & 0.9800 \\
$\mathrm{C} 7-\mathrm{S} 4$ & $1.714(3)$ & $\mathrm{C} 11-\mathrm{H} 11 \mathrm{~B}$ & 0.9800 \\
$\mathrm{O} 1-\mathrm{C} 3$ & $1.426(3)$ & $\mathrm{C} 11-\mathrm{H} 11 \mathrm{C}$ & 0.9800 \\
$\mathrm{O} 1-\mathrm{H} 1 \mathrm{O}$ & $0.843(10)$ & $\mathrm{C} 12-\mathrm{H} 12 \mathrm{~A}$ & 0.9800 \\
$\mathrm{O} 2-\mathrm{C} 9$ & $1.414(3)$ & $\mathrm{C} 12-\mathrm{H} 12 \mathrm{~B}$ & 0.9800 \\
$\mathrm{O} 2-\mathrm{H} 2 \mathrm{O}$ & $0.837(10)$ & $\mathrm{C} 12-\mathrm{H} 12 \mathrm{C}$ & 0.9800
\end{tabular}




\begin{tabular}{|c|c|c|c|}
\hline $\mathrm{N} 1-\mathrm{C} 1$ & $1.335(3)$ & $\mathrm{C} 13-\mathrm{C} 14$ & $1.381(4)$ \\
\hline $\mathrm{N} 1-\mathrm{C} 2$ & $1.471(3)$ & $\mathrm{C} 13-\mathrm{H} 13$ & 0.9500 \\
\hline $\mathrm{N} 1-\mathrm{C} 4$ & $1.486(3)$ & $\mathrm{C} 14-\mathrm{C} 15$ & $1.392(4)$ \\
\hline $\mathrm{N} 2-\mathrm{C} 7$ & $1.343(3)$ & $\mathrm{C} 14-\mathrm{H} 14$ & 0.9500 \\
\hline $\mathrm{N} 2-\mathrm{C} 8$ & $1.474(3)$ & $\mathrm{C} 15-\mathrm{C} 16$ & $1.396(4)$ \\
\hline $\mathrm{N} 2-\mathrm{C} 10$ & $1.483(3)$ & $\mathrm{C} 15-\mathrm{C} 18$ & $1.486(4)$ \\
\hline $\mathrm{N} 3-\mathrm{C} 13$ & $1.341(3)$ & $\mathrm{C} 16-\mathrm{C} 17$ & $1.378(4)$ \\
\hline N3- 177 & $1.347(3)$ & $\mathrm{C} 16-\mathrm{H} 16$ & 0.9500 \\
\hline $\mathrm{N} 4-\mathrm{C} 20$ & $1.335(4)$ & C17-H17 & 0.9500 \\
\hline $\mathrm{N} 4-\mathrm{C} 21$ & $1.338(3)$ & $\mathrm{C} 18-\mathrm{C} 22$ & $1.388(4)$ \\
\hline $\mathrm{N} 5-\mathrm{C} 23$ & $1.331(4)$ & $\mathrm{C} 18-\mathrm{C} 19$ & $1.395(4)$ \\
\hline N5-C27 & $1.337(4)$ & $\mathrm{C} 19-\mathrm{C} 20$ & $1.381(4)$ \\
\hline $\mathrm{C} 2-\mathrm{C} 3$ & $1.520(4)$ & C19-H19 & 0.9500 \\
\hline $\mathrm{C} 2-\mathrm{H} 2 \mathrm{~A}$ & 0.9900 & $\mathrm{C} 20-\mathrm{H} 20$ & 0.9500 \\
\hline $\mathrm{C} 2-\mathrm{H} 2 \mathrm{~B}$ & 0.9900 & $\mathrm{C} 21-\mathrm{C} 22$ & $1.377(4)$ \\
\hline $\mathrm{C} 3-\mathrm{H} 3 \mathrm{~A}$ & 0.9900 & $\mathrm{C} 21-\mathrm{H} 21$ & 0.9500 \\
\hline C $3-\mathrm{H} 3 \mathrm{~B}$ & 0.9900 & $\mathrm{C} 22-\mathrm{H} 22$ & 0.9500 \\
\hline $\mathrm{C} 4-\mathrm{C} 5$ & $1.519(4)$ & $\mathrm{C} 23-\mathrm{C} 24$ & $1.391(4)$ \\
\hline $\mathrm{C} 4-\mathrm{C} 6$ & $1.527(4)$ & $\mathrm{C} 23-\mathrm{H} 23$ & 0.9500 \\
\hline $\mathrm{C} 4-\mathrm{H} 4$ & 1.0000 & $\mathrm{C} 24-\mathrm{C} 25$ & $1.393(4)$ \\
\hline $\mathrm{C} 5-\mathrm{H} 5 \mathrm{~A}$ & 0.9800 & $\mathrm{C} 24-\mathrm{H} 24$ & 0.9500 \\
\hline C5-H5B & 0.9800 & $\mathrm{C} 25-\mathrm{C} 26$ & $1.388(4)$ \\
\hline $\mathrm{C} 5-\mathrm{H} 5 \mathrm{C}$ & 0.9800 & $\mathrm{C} 25-\mathrm{C} 25^{\mathrm{i}}$ & $1.489(5)$ \\
\hline C6-H6A & 0.9800 & $\mathrm{C} 26-\mathrm{C} 27$ & $1.377(4)$ \\
\hline C6-H6B & 0.9800 & $\mathrm{C} 26-\mathrm{H} 26$ & 0.9500 \\
\hline C6- $\mathrm{H} 6 \mathrm{C}$ & 0.9800 & $\mathrm{C} 27-\mathrm{H} 27$ & 0.9500 \\
\hline $\mathrm{C} 8-\mathrm{C} 9$ & $1.512(4)$ & & \\
\hline $\mathrm{N} 3-\mathrm{Zn}-\mathrm{S} 1$ & $120.49(7)$ & $\mathrm{O} 2-\mathrm{C} 9-\mathrm{C} 8$ & $107.2(2)$ \\
\hline N3-Zn-S3 & $115.32(7)$ & $\mathrm{O} 2-\mathrm{C} 9-\mathrm{H} 9 \mathrm{~A}$ & 110.3 \\
\hline $\mathrm{S} 1-\mathrm{Zn}-\mathrm{S} 3$ & $124.19(3)$ & $\mathrm{C} 8-\mathrm{C} 9-\mathrm{H} 9 \mathrm{~A}$ & 110.3 \\
\hline $\mathrm{N} 3-\mathrm{Zn}-\mathrm{S} 2$ & $97.53(6)$ & $\mathrm{O} 2-\mathrm{C} 9-\mathrm{H} 9 \mathrm{~B}$ & 110.3 \\
\hline $\mathrm{S} 1-\mathrm{Zn}-\mathrm{S} 2$ & $73.73(3)$ & $\mathrm{C} 8-\mathrm{C} 9-\mathrm{H} 9 \mathrm{~B}$ & 110.3 \\
\hline $\mathrm{S} 3-\mathrm{Zn}-\mathrm{S} 2$ & $99.76(3)$ & $\mathrm{H} 9 \mathrm{~A}-\mathrm{C} 9-\mathrm{H} 9 \mathrm{~B}$ & 108.5 \\
\hline $\mathrm{N} 3-\mathrm{Zn}-\mathrm{S} 4$ & $99.60(6)$ & $\mathrm{N} 2-\mathrm{C} 10-\mathrm{C} 11$ & $109.8(2)$ \\
\hline $\mathrm{S} 1-\mathrm{Zn}-\mathrm{S} 4$ & $97.06(3)$ & $\mathrm{N} 2-\mathrm{C} 10-\mathrm{C} 12$ & $112.1(2)$ \\
\hline $\mathrm{S} 3-\mathrm{Zn}-\mathrm{S} 4$ & $73.12(3)$ & $\mathrm{C} 11-\mathrm{C} 10-\mathrm{C} 12$ & $111.9(2)$ \\
\hline $\mathrm{S} 2-\mathrm{Zn}-\mathrm{S} 4$ & $162.87(3)$ & $\mathrm{N} 2-\mathrm{C} 10-\mathrm{H} 10$ & 107.6 \\
\hline $\mathrm{C} 1-\mathrm{S} 1-\mathrm{Zn}$ & $87.38(9)$ & $\mathrm{C} 11-\mathrm{C} 10-\mathrm{H} 10$ & 107.6 \\
\hline $\mathrm{C} 1-\mathrm{S} 2-\mathrm{Zn}$ & $82.03(9)$ & $\mathrm{C} 12-\mathrm{C} 10-\mathrm{H} 10$ & 107.6 \\
\hline $\mathrm{C} 7-\mathrm{S} 3-\mathrm{Zn}$ & $88.01(10)$ & $\mathrm{C} 10-\mathrm{C} 11-\mathrm{H} 11 \mathrm{~A}$ & 109.5 \\
\hline $\mathrm{C} 7-\mathrm{S} 4-\mathrm{Zn}$ & $81.07(10)$ & $\mathrm{C} 10-\mathrm{C} 11-\mathrm{H} 11 \mathrm{~B}$ & 109.5 \\
\hline $\mathrm{C} 3-\mathrm{O} 1-\mathrm{H} 1 \mathrm{O}$ & $106(2)$ & $\mathrm{H} 11 \mathrm{~A}-\mathrm{C} 11-\mathrm{H} 11 \mathrm{~B}$ & 109.5 \\
\hline $\mathrm{C} 9-\mathrm{O} 2-\mathrm{H} 2 \mathrm{O}$ & $105(2)$ & $\mathrm{C} 10-\mathrm{C} 11-\mathrm{H} 11 \mathrm{C}$ & 109.5 \\
\hline $\mathrm{C} 1-\mathrm{N} 1-\mathrm{C} 2$ & $120.0(2)$ & $\mathrm{H} 11 \mathrm{~A}-\mathrm{C} 11-\mathrm{H} 11 \mathrm{C}$ & 109.5 \\
\hline $\mathrm{C} 1-\mathrm{N} 1-\mathrm{C} 4$ & $121.0(2)$ & $\mathrm{H} 11 \mathrm{~B}-\mathrm{C} 11-\mathrm{H} 11 \mathrm{C}$ & 109.5 \\
\hline $\mathrm{C} 2-\mathrm{N} 1-\mathrm{C} 4$ & $118.8(2)$ & $\mathrm{C} 10-\mathrm{C} 12-\mathrm{H} 12 \mathrm{~A}$ & 109.5 \\
\hline $\mathrm{C} 7-\mathrm{N} 2-\mathrm{C} 8$ & $120.5(2)$ & $\mathrm{C} 10-\mathrm{C} 12-\mathrm{H} 12 \mathrm{~B}$ & 109.5 \\
\hline
\end{tabular}


$\mathrm{C} 7-\mathrm{N} 2-\mathrm{C} 10$

$\mathrm{C} 8-\mathrm{N} 2-\mathrm{C} 10$

$\mathrm{C} 13-\mathrm{N} 3-\mathrm{C} 17$

$\mathrm{C} 13-\mathrm{N} 3-\mathrm{Zn}$

C17-N3-Zn

$\mathrm{C} 20-\mathrm{N} 4-\mathrm{C} 21$

$\mathrm{C} 23-\mathrm{N} 5-\mathrm{C} 27$

$\mathrm{N} 1-\mathrm{C} 1-\mathrm{S} 2$

$\mathrm{N} 1-\mathrm{C} 1-\mathrm{S} 1$

$\mathrm{S} 2-\mathrm{C} 1-\mathrm{S} 1$

$\mathrm{N} 1-\mathrm{C} 2-\mathrm{C} 3$

$\mathrm{N} 1-\mathrm{C} 2-\mathrm{H} 2 \mathrm{~A}$

$\mathrm{C} 3-\mathrm{C} 2-\mathrm{H} 2 \mathrm{~A}$

$\mathrm{N} 1-\mathrm{C} 2-\mathrm{H} 2 \mathrm{~B}$

$\mathrm{C} 3-\mathrm{C} 2-\mathrm{H} 2 \mathrm{~B}$

$\mathrm{H} 2 \mathrm{~A}-\mathrm{C} 2-\mathrm{H} 2 \mathrm{~B}$

$\mathrm{O} 1-\mathrm{C} 3-\mathrm{C} 2$

$\mathrm{O} 1-\mathrm{C} 3-\mathrm{H} 3 \mathrm{~A}$

$\mathrm{C} 2-\mathrm{C} 3-\mathrm{H} 3 \mathrm{~A}$

$\mathrm{O} 1-\mathrm{C} 3-\mathrm{H} 3 \mathrm{~B}$

$\mathrm{C} 2-\mathrm{C} 3-\mathrm{H} 3 \mathrm{~B}$

$\mathrm{H} 3 \mathrm{~A}-\mathrm{C} 3-\mathrm{H} 3 \mathrm{~B}$

$\mathrm{N} 1-\mathrm{C} 4-\mathrm{C} 5$

$\mathrm{N} 1-\mathrm{C} 4-\mathrm{C} 6$

$\mathrm{C} 5-\mathrm{C} 4-\mathrm{C} 6$

$\mathrm{N} 1-\mathrm{C} 4-\mathrm{H} 4$

$\mathrm{C} 5-\mathrm{C} 4-\mathrm{H} 4$

$\mathrm{C} 6-\mathrm{C} 4-\mathrm{H} 4$

$\mathrm{C} 4-\mathrm{C} 5-\mathrm{H} 5 \mathrm{~A}$

$\mathrm{C} 4-\mathrm{C} 5-\mathrm{H} 5 \mathrm{~B}$

$\mathrm{H} 5 \mathrm{~A}-\mathrm{C} 5-\mathrm{H} 5 \mathrm{~B}$

$\mathrm{C} 4-\mathrm{C} 5-\mathrm{H} 5 \mathrm{C}$

$\mathrm{H} 5 \mathrm{~A}-\mathrm{C} 5-\mathrm{H} 5 \mathrm{C}$

$\mathrm{H} 5 \mathrm{~B}-\mathrm{C} 5-\mathrm{H} 5 \mathrm{C}$

C4- 6 6- $-\mathrm{H} 6 \mathrm{~A}$

$\mathrm{C} 4-\mathrm{C} 6-\mathrm{H} 6 \mathrm{~B}$

H6A-C6-H6B

$\mathrm{C} 4-\mathrm{C} 6-\mathrm{H} 6 \mathrm{C}$

$\mathrm{H} 6 \mathrm{~A}-\mathrm{C} 6-\mathrm{H} 6 \mathrm{C}$

$\mathrm{H} 6 \mathrm{~B}-\mathrm{C} 6-\mathrm{H} 6 \mathrm{C}$

$\mathrm{N} 2-\mathrm{C} 7-\mathrm{S} 4$

$\mathrm{N} 2-\mathrm{C} 7-\mathrm{S} 3$

$\mathrm{S} 4-\mathrm{C} 7-\mathrm{S} 3$

$\mathrm{N} 2-\mathrm{C} 8-\mathrm{C} 9$

$\mathrm{N} 2-\mathrm{C} 8-\mathrm{H} 8 \mathrm{~A}$

$\mathrm{C} 9-\mathrm{C} 8-\mathrm{H} 8 \mathrm{~A}$

$\mathrm{N} 2-\mathrm{C} 8-\mathrm{H} 8 \mathrm{~B}$

$\mathrm{C} 9-\mathrm{C} 8-\mathrm{H} 8 \mathrm{~B}$
$121.3(2)$

117.7 (2)

$117.0(2)$

$121.84(18)$

$121.11(17)$

116.7 (2)

$115.3(3)$

$122.3(2)$

$120.8(2)$

$116.84(15)$

111.0 (2)

109.4

109.4

109.4

109.4

108.0

109.4 (2)

109.8

109.8

109.8

109.8

108.2

109.9 (2)

$112.4(2)$

$111.8(2)$

107.5

107.5

107.5

109.5

109.5

109.5

109.5

109.5

109.5

109.5

109.5

109.5

109.5

109.5

109.5

122.3 (2)

$120.0(2)$

117.69 (16)

$112.2(2)$

109.2

109.2

109.2

109.2
$\mathrm{H} 12 \mathrm{~A}-\mathrm{C} 12-\mathrm{H} 12 \mathrm{~B}$

$\mathrm{C} 10-\mathrm{C} 12-\mathrm{H} 12 \mathrm{C}$

$\mathrm{H} 12 \mathrm{~A}-\mathrm{C} 12-\mathrm{H} 12 \mathrm{C}$

$\mathrm{H} 12 \mathrm{~B}-\mathrm{C} 12-\mathrm{H} 12 \mathrm{C}$

N3-C13-C14

N3- $13-\mathrm{H} 13$

$\mathrm{C} 14-\mathrm{C} 13-\mathrm{H} 13$

$\mathrm{C} 13-\mathrm{C} 14-\mathrm{C} 15$

C13-C14-H14

C15-C14-H14

C14-C15-C16

$\mathrm{C} 14-\mathrm{C} 15-\mathrm{C} 18$

C16-C15-C18

C17-C16-C15

C17-C16-H16

C15-C16-H16

N3-C17-C16

N3- 17 - 17

C16-C17-H17

C22-C18-C19

$\mathrm{C} 22-\mathrm{C} 18-\mathrm{C} 15$

$\mathrm{C} 19-\mathrm{C} 18-\mathrm{C} 15$

$\mathrm{C} 20-\mathrm{C} 19-\mathrm{C} 18$

$\mathrm{C} 20-\mathrm{C} 19-\mathrm{H} 19$

$\mathrm{C} 18-\mathrm{C} 19-\mathrm{H} 19$

N4-C20-C19

$\mathrm{N} 4-\mathrm{C} 20-\mathrm{H} 20$

$\mathrm{C} 19-\mathrm{C} 20-\mathrm{H} 20$

N4-C21-C22

$\mathrm{N} 4-\mathrm{C} 21-\mathrm{H} 21$

$\mathrm{C} 22-\mathrm{C} 21-\mathrm{H} 21$

$\mathrm{C} 21-\mathrm{C} 22-\mathrm{C} 18$

$\mathrm{C} 21-\mathrm{C} 22-\mathrm{H} 22$

$\mathrm{C} 18-\mathrm{C} 22-\mathrm{H} 22$

$\mathrm{N} 5-\mathrm{C} 23-\mathrm{C} 24$

$\mathrm{N} 5-\mathrm{C} 23-\mathrm{H} 23$

$\mathrm{C} 24-\mathrm{C} 23-\mathrm{H} 23$

$\mathrm{C} 23-\mathrm{C} 24-\mathrm{C} 25$

$\mathrm{C} 23-\mathrm{C} 24-\mathrm{H} 24$

$\mathrm{C} 25-\mathrm{C} 24-\mathrm{H} 24$

$\mathrm{C} 26-\mathrm{C} 25-\mathrm{C} 24$

$\mathrm{C} 26-\mathrm{C} 25-\mathrm{C} 25^{\mathrm{i}}$

$\mathrm{C} 24-\mathrm{C} 25-\mathrm{C} 25^{\mathrm{i}}$

$\mathrm{C} 27-\mathrm{C} 26-\mathrm{C} 25$

$\mathrm{C} 27-\mathrm{C} 26-\mathrm{H} 26$

$\mathrm{C} 25-\mathrm{C} 26-\mathrm{H} 26$

$\mathrm{N} 5-\mathrm{C} 27-\mathrm{C} 26$

N5-C27-H27
109.5

109.5

109.5

109.5

$123.2(2)$

118.4

118.4

120.0 (2)

120.0

120.0

116.8 (2)

$121.8(2)$

$121.4(2)$

119.8 (2)

120.1

120.1

$123.2(2)$

118.4

118.4

117.4 (2)

120.6 (2)

121.9 (2)

119.4 (3)

120.3

120.3

123.4 (3)

118.3

118.3

124.2 (3)

117.9

117.9

118.9 (3)

120.6

120.6

124.5 (3)

117.8

117.8

119.4 (3)

120.3

120.3

116.3 (2)

122.2 (3)

121.5 (3)

119.9 (3)

120.1

120.1

124.7 (3)

117.7 


$\mathrm{H} 8 \mathrm{~A}-\mathrm{C} 8-\mathrm{H} 8 \mathrm{~B}$
$\mathrm{C} 2-\mathrm{N} 1-\mathrm{C} 1-\mathrm{S} 2$
$\mathrm{C} 4-\mathrm{N} 1-\mathrm{C} 1-\mathrm{S} 2$
$\mathrm{C} 2-\mathrm{N} 1-\mathrm{C} 1-\mathrm{S} 1$
$\mathrm{C} 4-\mathrm{N} 1-\mathrm{C} 1-\mathrm{S} 1$
$\mathrm{Zn}-\mathrm{S} 2-\mathrm{C} 1-\mathrm{N} 1$
$\mathrm{Zn}-\mathrm{S} 2-\mathrm{C} 1-\mathrm{S} 1$
$\mathrm{Zn}-\mathrm{S} 1-\mathrm{C} 1-\mathrm{N} 1$
$\mathrm{Zn}-\mathrm{S} 1-\mathrm{C} 1-\mathrm{S} 2$
$\mathrm{C} 1-\mathrm{N} 1-\mathrm{C} 2-\mathrm{C} 3$
$\mathrm{C} 4-\mathrm{N} 1-\mathrm{C} 2-\mathrm{C} 3$
$\mathrm{~N} 1-\mathrm{C} 2-\mathrm{C} 3-\mathrm{O} 1$
$\mathrm{C} 1-\mathrm{N} 1-\mathrm{C} 4-\mathrm{C} 5$
$\mathrm{C} 2-\mathrm{N} 1-\mathrm{C} 4-\mathrm{C} 5$
$\mathrm{C} 1-\mathrm{N} 1-\mathrm{C} 4-\mathrm{C} 6$
$\mathrm{C} 2-\mathrm{N} 1-\mathrm{C} 4-\mathrm{C} 6$
$\mathrm{C} 8-\mathrm{N} 2-\mathrm{C} 7-\mathrm{S} 4$
$\mathrm{C} 10-\mathrm{N} 2-\mathrm{C} 7-\mathrm{S} 4$
$\mathrm{C} 8-\mathrm{N} 2-\mathrm{C} 7-\mathrm{S} 3$
$\mathrm{C} 10-\mathrm{N} 2-\mathrm{C} 7-\mathrm{S} 3$
$\mathrm{Zn}-\mathrm{S} 4-\mathrm{C} 7-\mathrm{N} 2$
$\mathrm{Zn}-\mathrm{S} 4-\mathrm{C} 7-\mathrm{S} 3$
$\mathrm{Zn}-\mathrm{S} 3-\mathrm{C} 7-\mathrm{N} 2$
$\mathrm{Zn}-\mathrm{S} 3-\mathrm{C} 7-\mathrm{S} 4$
$\mathrm{C} 7-\mathrm{N} 2-\mathrm{C} 8-\mathrm{C} 9$
$\mathrm{C} 10-\mathrm{N} 2-\mathrm{C} 8-\mathrm{C} 9$
$\mathrm{~N} 2-\mathrm{C} 8-\mathrm{C} 9-\mathrm{O} 2$
$\mathrm{C} 7-\mathrm{N} 2-\mathrm{C} 10-\mathrm{C} 11$
$\mathrm{C} 8-\mathrm{N} 2-\mathrm{C} 10-\mathrm{C} 11$
$\mathrm{C} 7-\mathrm{N} 2-\mathrm{C} 10-\mathrm{C} 12$
$\mathrm{C} 8-\mathrm{N} 2-\mathrm{C} 10-\mathrm{C} 12$

107.9

$-178.61(19)$

$-3.1(3)$

$1.6(3)$

$177.10(19)$

$-178.6(2)$

$1.23(13)$

$178.5(2)$

$-1.31(14)$

$-83.1(3)$

$101.3(3)$

$177.3(2)$

$-93.7(3)$

$81.8(3)$

$141.0(3)$

$-43.5(3)$

$-178.27(18)$

$-6.7(3)$

$2.4(3)$

$173.96(19)$

$-176.4(2)$

$2.93(13)$

$176.2(2)$

$-3.19(14)$

$-86.4(3)$

$101.7(3)$

$-176.9(2)$

$-94.0(3)$

77.8 (3)

140.9 (3)

-47.3 (3)
$\mathrm{C} 26-\mathrm{C} 27-\mathrm{H} 27$

117.7

$1.4(4)$

-176.2 (2)

-0.5 (4)

$-1.3(4)$

177.0 (3)

2.1 (4)

-176.2 (3)

-0.5 (4)

$177.1(2)$

-1.2 (4)

-150.2 (3)

28.0 (4)

27.9 (4)

-153.9 (3)

0.2 (4)

-178.0 (3)

-0.4 (4)

0.2 (5)

0.4 (5)

$-0.1(5)$

-0.2 (4)

177.9 (3)

$1.1(5)$

-0.4 (5)

-0.1 (4)

178.3 (3)

-0.1 (4)

-178.5 (3)

$-1.3(5)$

0.8 (5)

Symmetry code: (i) $-x,-y+2,-z$.

Hydrogen-bond geometry $\left(\AA,{ }^{\circ}\right)$

$\mathrm{Cg} 1$ is the centroid of the $\mathrm{Cd} / \mathrm{S} 3 / \mathrm{S} 4 / \mathrm{C} 7$ chelate ring.

\begin{tabular}{lllll}
\hline$D-\mathrm{H} \cdots A$ & $D-\mathrm{H}$ & $\mathrm{H} \cdots A$ & $D \cdots A$ & $D-\mathrm{H} \cdots A$ \\
\hline $\mathrm{O} 1-\mathrm{H} 1 O \cdots \mathrm{N} 4^{4 i}$ & $0.85(2)$ & $1.85(2)$ & $2.697(3)$ & $179(3)$ \\
$\mathrm{O} 2-\mathrm{H} 2 O \cdots \mathrm{O} 1^{i i i}$ & $0.84(2)$ & $1.88(2)$ & $2.718(3)$ & $176(4)$ \\
$\mathrm{C} 4-\mathrm{H} 4 \cdots \mathrm{S} 2^{\mathrm{iv}}$ & 1.00 & 2.67 & $3.515(3)$ & 142 \\
$\mathrm{C} 22-\mathrm{H} 22 \cdots \mathrm{O} 2^{\mathrm{v}}$ & 0.95 & 2.36 & $3.300(3)$ & 170 \\
$\mathrm{C} 26-\mathrm{H} 26 \cdots \mathrm{Cg}^{\mathrm{ii}}$ & 0.95 & 3.04 & $3.7943(15)$ & 138 \\
\hline
\end{tabular}

Symmetry codes: (ii) $-x, y-1,-z+1 / 2$; (iii) $x+1 / 2, y+1 / 2, z$; (iv) $x+1 / 2, y+3 / 2, z$; (v) $x,-y, z-1 / 2$; (vi) $x, y+1, z$. 
Crystal data

$\left[\mathrm{Cd}\left(\mathrm{C}_{6} \mathrm{H}_{12} \mathrm{NOS}_{2}\right)_{2}\left(\mathrm{C}_{10} \mathrm{H}_{8} \mathrm{~N}_{2}\right)\right] \cdot 0.5 \mathrm{C}_{10} \mathrm{H}_{8} \mathrm{~N}_{2}$

$M_{r}=703.25$

Monoclinic, $C 2 / c$

$a=22.7028(12) \AA$

$b=11.5950$ (6) $\AA$

$c=24.8196(13) \AA$

$\beta=103.385(1)^{\circ}$

$V=6356.0(6) \AA^{3}$

$Z=8$

Data collection

Bruker SMART APEX CCD

diffractometer

Radiation source: fine-focus sealed tube

Graphite monochromator

$\omega$ scans

Absorption correction: multi-scan

(SADABS; Sheldrick, 1996)

$T_{\min }=0.962, T_{\max }=0.971$

Refinement

Refinement on $F^{2}$

Least-squares matrix: full

$R\left[F^{2}>2 \sigma\left(F^{2}\right)\right]=0.019$

$w R\left(F^{2}\right)=0.049$

$S=0.99$

7847 reflections

358 parameters

2 restraints
$F(000)=2888$

$D_{\mathrm{x}}=1.470 \mathrm{Mg} \mathrm{m}^{-3}$

Mo $K \alpha$ radiation, $\lambda=0.71073 \AA$

Cell parameters from 24754 reflections

$\theta=2.2-28.3^{\circ}$

$\mu=0.98 \mathrm{~mm}^{-1}$

$T=100 \mathrm{~K}$

Block, yellow

$0.04 \times 0.04 \times 0.03 \mathrm{~mm}$
41284 measured reflections

7847 independent reflections

7204 reflections with $I>2 \sigma(I)$

$R_{\text {int }}=0.023$

$\theta_{\text {max }}=28.3^{\circ}, \theta_{\min }=1.7^{\circ}$

$h=-30 \rightarrow 30$

$k=-15 \rightarrow 15$

$l=-33 \rightarrow 33$

Primary atom site location: structure-invariant direct methods

Hydrogen site location: mixed

$\mathrm{H}$ atoms treated by a mixture of independent and constrained refinement

$w=1 /\left[\sigma^{2}\left(F_{0}^{2}\right)+(0.0249 P)^{2}+6.291 P\right]$ where $P=\left(F_{\mathrm{o}}^{2}+2 F_{\mathrm{c}}^{2}\right) / 3$

$(\Delta / \sigma)_{\max }=0.001$

$\Delta \rho_{\max }=0.46 \mathrm{e} \AA^{-3}$

$\Delta \rho_{\min }=-0.24 \mathrm{e} \AA^{-3}$

Special details

Geometry. All esds (except the esd in the dihedral angle between two 1.s. planes) are estimated using the full covariance matrix. The cell esds are taken into account individually in the estimation of esds in distances, angles and torsion angles; correlations between esds in cell parameters are only used when they are defined by crystal symmetry. An approximate (isotropic) treatment of cell esds is used for estimating esds involving l.s. planes.

Fractional atomic coordinates and isotropic or equivalent isotropic displacement parameters $\left(\AA^{2}\right)$

\begin{tabular}{lllll}
\hline & $x$ & $y$ & $z$ & $U_{\text {iso }}{ }^{*} / U_{\text {eq }}$ \\
\hline $\mathrm{Cd}$ & $0.04996(2)$ & $0.31263(2)$ & $0.16486(2)$ & $0.01723(3)$ \\
$\mathrm{S} 1$ & $-0.05744(2)$ & $0.24286(3)$ & $0.12217(2)$ & $0.01723(6)$ \\
$\mathrm{S} 2$ & $0.00085(2)$ & $0.43727(3)$ & $0.07636(2)$ & $0.01907(7)$ \\
$\mathrm{S} 3$ & $0.14726(2)$ & $0.23031(3)$ & $0.14448(2)$ & $0.02008(7)$ \\
$\mathrm{S} 4$ & $0.09406(2)$ & $0.14429(3)$ & $0.23619(2)$ & $0.02109(7)$ \\
$\mathrm{O} 1$ & $-0.20171(4)$ & $0.08734(8)$ & $0.00837(4)$ & $0.02070(19)$ \\
$\mathrm{H} 1 \mathrm{O}$ & $-0.1878(8)$ & $0.0408(13)$ & $0.0337(6)$ & $0.031^{*}$ \\
$\mathrm{O} 2$ & $0.26295(4)$ & $-0.02139(9)$ & $0.09436(4)$ & $0.0253(2)$ \\
$\mathrm{H} 2 \mathrm{O}$ & $0.2468(8)$ & $-0.0409(17)$ & $0.0620(5)$ & $0.038^{*}$
\end{tabular}




\begin{tabular}{|c|c|c|c|c|}
\hline N1 & $-0.10858(5)$ & $0.35363(9)$ & $0.02905(4)$ & $0.0151(2)$ \\
\hline N2 & $0.19087(5)$ & $0.05102(10)$ & $0.20849(4)$ & $0.0180(2)$ \\
\hline N3 & $0.06811(5)$ & $0.46557(9)$ & $0.22553(4)$ & $0.0166(2)$ \\
\hline N4 & $0.15540(6)$ & $0.92852(10)$ & $0.41323(5)$ & $0.0257(2)$ \\
\hline N5 & $0.02716(6)$ & $0.79642(11)$ & $0.11132(6)$ & $0.0294(3)$ \\
\hline $\mathrm{C} 1$ & $-0.05999(5)$ & $0.34592(10)$ & $0.07123(5)$ & $0.0146(2)$ \\
\hline $\mathrm{C} 2$ & $-0.16139(5)$ & $0.27871(11)$ & $0.02738(5)$ & $0.0169(2)$ \\
\hline $\mathrm{H} 2 \mathrm{~A}$ & -0.1980 & 0.3156 & 0.0045 & $0.020^{*}$ \\
\hline $\mathrm{H} 2 \mathrm{~B}$ & -0.1676 & 0.2693 & 0.0653 & $0.020^{*}$ \\
\hline $\mathrm{C} 3$ & $-0.15277(6)$ & $0.16054(11)$ & $0.00341(5)$ & $0.0189(2)$ \\
\hline $\mathrm{H} 3 \mathrm{~A}$ & -0.1516 & 0.1683 & -0.0360 & $0.023 *$ \\
\hline H3B & -0.1139 & 0.1266 & 0.0237 & $0.023 *$ \\
\hline $\mathrm{C} 4$ & $-0.11285(6)$ & $0.44310(11)$ & $-0.01475(5)$ & $0.0187(2)$ \\
\hline H4 & -0.0706 & 0.4674 & -0.0149 & $0.022 *$ \\
\hline C5 & $-0.14574(7)$ & $0.54876(13)$ & $-0.00028(7)$ & $0.0298(3)$ \\
\hline H5A & -0.1484 & 0.6072 & -0.0293 & $0.045^{*}$ \\
\hline H5B & -0.1866 & 0.5269 & 0.0025 & $0.045^{*}$ \\
\hline $\mathrm{H} 5 \mathrm{C}$ & -0.1234 & 0.5803 & 0.0352 & $0.045^{*}$ \\
\hline $\mathrm{C} 6$ & $-0.14156(7)$ & $0.39715(14)$ & $-0.07228(6)$ & $0.0269(3)$ \\
\hline H6A & -0.1434 & 0.4589 & -0.0996 & $0.040^{*}$ \\
\hline H6B & -0.1172 & 0.3332 & -0.0811 & $0.040^{*}$ \\
\hline H6C & -0.1826 & 0.3698 & -0.0732 & $0.040^{*}$ \\
\hline C7 & $0.14824(6)$ & $0.13339(11)$ & $0.19783(5)$ & $0.0176(2)$ \\
\hline $\mathrm{C} 8$ & $0.23758(6)$ & $0.04441(12)$ & $0.17638(5)$ & $0.0198(3)$ \\
\hline H8A & 0.2740 & 0.0063 & 0.1990 & $0.024 *$ \\
\hline H8B & 0.2491 & 0.1234 & 0.1677 & $0.024^{*}$ \\
\hline C9 & $0.21556(6)$ & $-0.02239(12)$ & $0.12279(6)$ & $0.0216(3)$ \\
\hline H9A & 0.2055 & -0.1027 & 0.1309 & $0.026^{*}$ \\
\hline H9B & 0.1788 & 0.0143 & 0.0999 & $0.026^{*}$ \\
\hline $\mathrm{C} 10$ & $0.19723(6)$ & $-0.02799(12)$ & $0.25659(6)$ & $0.0235(3)$ \\
\hline H10 & 0.1579 & -0.0276 & 0.2682 & $0.028 *$ \\
\hline C11 & $0.24587(8)$ & $0.01794(16)$ & $0.30508(6)$ & $0.0369(4)$ \\
\hline H11A & 0.2500 & -0.0343 & 0.3368 & $0.055^{*}$ \\
\hline H11B & 0.2846 & 0.0229 & 0.2941 & $0.055^{*}$ \\
\hline $\mathrm{H} 11 \mathrm{C}$ & 0.2342 & 0.0948 & 0.3154 & $0.055^{*}$ \\
\hline $\mathrm{C} 12$ & $0.20978(7)$ & $-0.15172(13)$ & $0.24188(7)$ & $0.0302(3)$ \\
\hline $\mathrm{H} 12 \mathrm{~A}$ & 0.2136 & -0.2008 & 0.2747 & $0.045^{*}$ \\
\hline H12B & 0.1763 & -0.1796 & 0.2124 & $0.045^{*}$ \\
\hline $\mathrm{H} 12 \mathrm{C}$ & 0.2475 & -0.1544 & 0.2291 & $0.045^{*}$ \\
\hline $\mathrm{C} 13$ & $0.06012(6)$ & $0.57559(11)$ & $0.20834(5)$ & $0.0184(2)$ \\
\hline H13 & 0.0437 & 0.5903 & 0.1702 & $0.022 *$ \\
\hline $\mathrm{C} 14$ & $0.07479(6)$ & $0.66804(11)$ & $0.24394(5)$ & $0.0182(2)$ \\
\hline H14 & 0.0688 & 0.7446 & 0.2301 & $0.022 *$ \\
\hline $\mathrm{C} 15$ & $0.09848(5)$ & $0.64880(11)$ & $0.30033(5)$ & $0.0153(2)$ \\
\hline $\mathrm{C} 16$ & $0.10526(6)$ & $0.53450(11)$ & $0.31809(5)$ & $0.0168(2)$ \\
\hline H16 & 0.1201 & 0.5173 & 0.3562 & $0.020^{*}$ \\
\hline $\mathrm{C} 17$ & $0.09027(6)$ & $0.44634(11)$ & $0.27985(5)$ & $0.0172(2)$ \\
\hline H17 & 0.0959 & 0.3689 & 0.2925 & $0.021^{*}$ \\
\hline
\end{tabular}




$\begin{array}{lllll}\text { C18 } & 0.11718(6) & 0.74550(11) & 0.33969(5) & 0.0167(2) \\ \text { C19 } & 0.08995(6) & 0.85379(12) & 0.33087(6) & 0.0226(3) \\ \text { H19 } & 0.0577 & 0.8671 & 0.2995 & 0.027^{*} \\ \text { C20 } & 0.11034(7) & 0.94170(12) & 0.36829(6) & 0.0263(3) \\ \text { H20 } & 0.0914 & 1.0150 & 0.3617 & 0.032^{*} \\ \text { C21 } & 0.18115(7) & 0.82418(12) & 0.42167(6) & 0.0241(3) \\ \text { H21 } & 0.2131 & 0.8134 & 0.4535 & 0.029^{*} \\ \text { C22 } & 0.16396(6) & 0.73140(11) & 0.38674(5) & 0.0196(2) \\ \text { H22 } & 0.1837 & 0.6590 & 0.3946 & 0.024^{*} \\ \text { C23 } & -0.00188(8) & 0.76757(13) & 0.05982(7) & 0.0323(3) \\ \text { H23 } & -0.0154 & 0.6902 & 0.0533 & 0.039^{*} \\ \text { C24 } & -0.01356(7) & 0.84325(12) & 0.01538(6) & 0.0288(3) \\ \text { H24 } & -0.0343 & 0.8172 & -0.0203 & 0.035^{*} \\ \text { C25 } & 0.00530(6) & 0.95788(11) & 0.02321(5) & 0.0188(2) \\ \text { C26 } & 0.03523(7) & 0.98828(12) & 0.07680(6) & 0.0258(3) \\ \text { H26 } & 0.0490 & 1.0651 & 0.0848 & 0.031^{*} \\ \text { C27 } & 0.04485(7) & 0.90635(13) & 0.11837(7) & 0.0299(3) \\ \text { H27 } & 0.0655 & 0.9299 & 0.1545 & 0.036^{*} \\ \end{array}$

Atomic displacement parameters $\left(\AA^{2}\right)$

\begin{tabular}{|c|c|c|c|c|c|c|}
\hline & $U^{11}$ & $U^{22}$ & $U^{33}$ & $U^{12}$ & $U^{13}$ & $U^{23}$ \\
\hline $\mathrm{Cd}$ & $0.01892(5)$ & $0.01605(5)$ & $0.01519(5)$ & $-0.00140(3)$ & $0.00083(3)$ & $-0.00184(3)$ \\
\hline $\mathrm{S} 1$ & $0.02100(15)$ & $0.01453(14)$ & $0.01510(14)$ & $-0.00512(11)$ & $0.00201(11)$ & $0.00145(11)$ \\
\hline $\mathrm{S} 2$ & $0.02016(15)$ & $0.01946(15)$ & $0.01593(14)$ & $-0.00819(12)$ & $0.00078(11)$ & $0.00279(11)$ \\
\hline S3 & $0.02200(15)$ & $0.02007(15)$ & $0.01891(15)$ & $-0.00138(12)$ & $0.00624(12)$ & $0.00193(12)$ \\
\hline S4 & $0.02419(16)$ & $0.02194(16)$ & $0.01883(15)$ & $0.00403(12)$ & $0.00847(12)$ & $0.00277(12)$ \\
\hline $\mathrm{O} 1$ & $0.0189(4)$ & $0.0182(4)$ & $0.0216(5)$ & $-0.0049(4)$ & $-0.0023(4)$ & $0.0050(4)$ \\
\hline $\mathrm{O} 2$ & $0.0196(5)$ & $0.0355(6)$ & $0.0214(5)$ & -0.0060 & $0.0062(4)$ & $-0.0109(4)$ \\
\hline N1 & $0.0155(5)$ & $0.0150(5)$ & $0.0151(5)$ & $-0.0024(4)$ & $0.0041(4)$ & $0.0010(4)$ \\
\hline N2 & $0.0174(5)$ & $0.0204(5)$ & $0.0158(5)$ & $-0.0001(4)$ & $0.0028(4)$ & $-0.0006(4)$ \\
\hline N3 & $0.0167(5)$ & $0.0170(5)$ & $0.0150(5)$ & $-0.0016(4)$ & $0.0016(4)$ & $-0.0009(4)$ \\
\hline N4 & $0.0340(7)$ & $0.0196(6)$ & $0.0230(6)$ & $-0.0005(5)$ & $0.0057(5)$ & $-0.0054(5)$ \\
\hline N5 & $0.0313(7)$ & $0.0235(6)$ & $0.0328(7)$ & $0.0015(5)$ & $0.0061(5)$ & $0.0050(5)$ \\
\hline $\mathrm{C} 1$ & $0.0170(6)$ & $0.0129(5)$ & $0.0146(5)$ & -0.0020 & $0.0052(4)$ & $-0.0016(4)$ \\
\hline $\mathrm{C} 2$ & $0.0135(5)$ & $0.0172(6)$ & $0.0198(6)$ & $-0.0028(4)$ & $0.0036(5)$ & $0.0001(5)$ \\
\hline C3 & $0.0172(6)$ & $0.0188(6)$ & $0.0192(6)$ & $-0.0040(5)$ & $0.0015(5)$ & $-0.0023(5)$ \\
\hline $\mathrm{C} 4$ & $0.0186(6)$ & $0.0207(6)$ & $0.0167(6)$ & $-0.0018(5)$ & $0.0036(5)$ & $0.0054(5)$ \\
\hline $\mathrm{C} 5$ & $0.0379(8)$ & $0.0205(7)$ & $0.0345(8)$ & $0.0032(6)$ & $0.0156(7)$ & $0.0084(6)$ \\
\hline C6 & $0.0281(7)$ & $0.0324(8)$ & $0.0175(6)$ & $-0.0029(6)$ & $-0.0002(5)$ & $0.0056(6)$ \\
\hline $\mathrm{C7}$ & $0.0188(6)$ & $0.0182(6)$ & $0.0146(5)$ & $-0.0037(5)$ & $0.0014(4)$ & $-0.0033(5)$ \\
\hline $\mathrm{C} 8$ & $0.0168(6)$ & $0.0228(6)$ & $0.0197(6)$ & $-0.0019(5)$ & $0.0038(5)$ & $-0.0047(5)$ \\
\hline C9 & $0.0192(6)$ & $0.0248(7)$ & $0.0211(6)$ & $-0.0047(5)$ & $0.0055(5)$ & $-0.0067(5)$ \\
\hline $\mathrm{C} 10$ & $0.0236(7)$ & $0.0269(7)$ & $0.0191(6)$ & $0.0044(5)$ & $0.0031(5)$ & $0.0046(5)$ \\
\hline C11 & $0.0408(9)$ & $0.0442(10)$ & $0.0199(7)$ & $0.0038(8)$ & $-0.0047(6)$ & $0.0004(7)$ \\
\hline C12 & $0.0320(8)$ & $0.0254(7)$ & $0.0336(8)$ & $0.0054(6)$ & $0.0087(6)$ & $0.0083(6)$ \\
\hline C13 & $0.0188(6)$ & $0.0203(6)$ & $0.0149(6)$ & $0.0009(5)$ & $0.0013(5)$ & $0.0010(5)$ \\
\hline C14 & $0.0192(6)$ & $0.0159(6)$ & $0.0182(6)$ & $0.0024(5)$ & $0.0018(5)$ & $0.0019(5)$ \\
\hline
\end{tabular}




$\begin{array}{lllllll}\mathrm{C} 15 & 0.0131(5) & 0.0164(6) & 0.0160(6) & 0.0000(4) & 0.0028(4) & -0.0020(4) \\ \mathrm{C} 16 & 0.0175(6) & 0.0187(6) & 0.0136(5) & -0.0006(5) & 0.0026(4) & 0.0011(4) \\ \mathrm{C} 17 & 0.0184(6) & 0.0158(6) & 0.0166(6) & -0.0018(5) & 0.0025(5) & 0.0015(5) \\ \mathrm{C} 18 & 0.0187(6) & 0.0158(6) & 0.0164(6) & -0.0008(5) & 0.0055(5) & -0.0009(5) \\ \mathrm{C} 19 & 0.0254(7) & 0.0199(6) & 0.0213(6) & 0.0050(5) & 0.0032(5) & -0.0009(5) \\ \mathrm{C} 20 & 0.0337(8) & 0.0170(6) & 0.0273(7) & 0.0053(6) & 0.0055(6) & -0.0029(5) \\ \mathrm{C} 21 & 0.0283(7) & 0.0224(7) & 0.0188(6) & -0.0006(5) & -0.0005(5) & -0.0034(5) \\ \mathrm{C} 22 & 0.0228(6) & 0.0166(6) & 0.0188(6) & 0.0019(5) & 0.0036(5) & -0.0012(5) \\ \mathrm{C} 23 & 0.0436(9) & 0.0152(6) & 0.0365(8) & -0.0051(6) & 0.0062(7) & 0.0002(6) \\ \mathrm{C} 24 & 0.0392(8) & 0.0171(6) & 0.0284(7) & -0.0059(6) & 0.0045(6) & -0.0032(6) \\ \mathrm{C} 25 & 0.0169(6) & 0.0146(6) & 0.0256(7) & 0.0011(5) & 0.0067(5) & -0.0018(5) \\ \mathrm{C} 26 & 0.0272(7) & 0.0182(6) & 0.0291(7) & -0.0026(5) & 0.0010(6) & -0.0026(5) \\ \mathrm{C} 27 & 0.0328(8) & 0.0253(7) & 0.0284(7) & -0.0006(6) & 0.0003(6) & 0.0005(6) \\ & & & & & & \end{array}$

Geometric parameters $\left(\AA,{ }^{\circ}\right)$

\begin{tabular}{|c|c|c|c|}
\hline $\mathrm{Cd}-\mathrm{N} 3$ & $2.3011(11)$ & $\mathrm{C} 8-\mathrm{H} 8 \mathrm{~A}$ & 0.9900 \\
\hline $\mathrm{Cd}-\mathrm{S} 1$ & $2.5547(3)$ & $\mathrm{C} 8-\mathrm{H} 8 \mathrm{~B}$ & 0.9900 \\
\hline $\mathrm{Cd}-\mathrm{S} 2$ & $2.6500(3)$ & C9-H9A & 0.9900 \\
\hline $\mathrm{Cd}-\mathrm{S} 3$ & $2.5620(4)$ & $\mathrm{C} 9-\mathrm{H} 9 \mathrm{~B}$ & 0.9900 \\
\hline $\mathrm{Cd}-\mathrm{S} 4$ & $2.6696(4)$ & $\mathrm{C} 10-\mathrm{C} 12$ & $1.523(2)$ \\
\hline $\mathrm{C} 1-\mathrm{S} 1$ & $1.7310(12)$ & $\mathrm{C} 10-\mathrm{C} 11$ & $1.528(2)$ \\
\hline $\mathrm{C} 1-\mathrm{S} 2$ & $1.7218(12)$ & $\mathrm{C} 10-\mathrm{H} 10$ & 1.0000 \\
\hline C7-S3 & $1.7328(13)$ & $\mathrm{C} 11-\mathrm{H} 11 \mathrm{~A}$ & 0.9800 \\
\hline $\mathrm{C} 7-\mathrm{S} 4$ & $1.7257(13)$ & $\mathrm{C} 11-\mathrm{H} 11 \mathrm{~B}$ & 0.9800 \\
\hline $\mathrm{O} 1-\mathrm{C} 3$ & $1.4259(15)$ & $\mathrm{C} 11-\mathrm{H} 11 \mathrm{C}$ & 0.9800 \\
\hline $\mathrm{O} 1-\mathrm{H} 1 \mathrm{O}$ & $0.833(9)$ & $\mathrm{C} 12-\mathrm{H} 12 \mathrm{~A}$ & 0.9800 \\
\hline $\mathrm{O} 2-\mathrm{C} 9$ & $1.4168(16)$ & $\mathrm{C} 12-\mathrm{H} 12 \mathrm{~B}$ & 0.9800 \\
\hline $\mathrm{O} 2-\mathrm{H} 2 \mathrm{O}$ & $0.832(9)$ & $\mathrm{C} 12-\mathrm{H} 12 \mathrm{C}$ & 0.9800 \\
\hline $\mathrm{N} 1-\mathrm{C} 1$ & $1.3364(16)$ & $\mathrm{C} 13-\mathrm{C} 14$ & $1.3801(18)$ \\
\hline $\mathrm{N} 1-\mathrm{C} 2$ & $1.4733(15)$ & $\mathrm{C} 13-\mathrm{H} 13$ & 0.9500 \\
\hline $\mathrm{N} 1-\mathrm{C} 4$ & $1.4895(16)$ & $\mathrm{C} 14-\mathrm{C} 15$ & $1.3960(17)$ \\
\hline $\mathrm{N} 2-\mathrm{C} 7$ & $1.3416(17)$ & $\mathrm{C} 14-\mathrm{H} 14$ & 0.9500 \\
\hline $\mathrm{N} 2-\mathrm{C} 8$ & $1.4688(16)$ & $\mathrm{C} 15-\mathrm{C} 16$ & $1.3940(17)$ \\
\hline $\mathrm{N} 2-\mathrm{C} 10$ & $1.4851(17)$ & $\mathrm{C} 15-\mathrm{C} 18$ & $1.4832(17)$ \\
\hline $\mathrm{N} 3-\mathrm{C} 13$ & $1.3441(16)$ & $\mathrm{C} 16-\mathrm{C} 17$ & $1.3827(17)$ \\
\hline N3-C17 & $1.3441(16)$ & $\mathrm{C} 16-\mathrm{H} 16$ & 0.9500 \\
\hline $\mathrm{N} 4-\mathrm{C} 20$ & $1.3363(19)$ & C17-H17 & 0.9500 \\
\hline $\mathrm{N} 4-\mathrm{C} 21$ & $1.3389(18)$ & $\mathrm{C} 18-\mathrm{C} 19$ & $1.3942(18)$ \\
\hline N5-C27 & $1.3356(19)$ & $\mathrm{C} 18-\mathrm{C} 22$ & $1.3944(18)$ \\
\hline $\mathrm{N} 5-\mathrm{C} 23$ & $1.338(2)$ & $\mathrm{C} 19-\mathrm{C} 20$ & $1.3846(19)$ \\
\hline $\mathrm{C} 2-\mathrm{C} 3$ & $1.5242(18)$ & C19-H19 & 0.9500 \\
\hline $\mathrm{C} 2-\mathrm{H} 2 \mathrm{~A}$ & 0.9900 & $\mathrm{C} 20-\mathrm{H} 20$ & 0.9500 \\
\hline $\mathrm{C} 2-\mathrm{H} 2 \mathrm{~B}$ & 0.9900 & $\mathrm{C} 21-\mathrm{C} 22$ & $1.3801(18)$ \\
\hline $\mathrm{C} 3-\mathrm{H} 3 \mathrm{~A}$ & 0.9900 & $\mathrm{C} 21-\mathrm{H} 21$ & 0.9500 \\
\hline C $3-\mathrm{H} 3 \mathrm{~B}$ & 0.9900 & $\mathrm{C} 22-\mathrm{H} 22$ & 0.9500 \\
\hline $\mathrm{C} 4-\mathrm{C} 5$ & $1.520(2)$ & $\mathrm{C} 23-\mathrm{C} 24$ & $1.386(2)$ \\
\hline $\mathrm{C} 4-\mathrm{C} 6$ & $1.5220(19)$ & $\mathrm{C} 23-\mathrm{H} 23$ & 0.9500 \\
\hline
\end{tabular}




\begin{tabular}{|c|c|c|c|}
\hline $\mathrm{C} 4-\mathrm{H} 4$ & 1.0000 & $\mathrm{C} 24-\mathrm{C} 25$ & $1.3960(18)$ \\
\hline $\mathrm{C} 5-\mathrm{H} 5 \mathrm{~A}$ & 0.9800 & $\mathrm{C} 24-\mathrm{H} 24$ & 0.9500 \\
\hline C5-H5B & 0.9800 & $\mathrm{C} 25-\mathrm{C} 26$ & 1.3917 (19) \\
\hline $\mathrm{C} 5-\mathrm{H} 5 \mathrm{C}$ & 0.9800 & $\mathrm{C} 25-\mathrm{C} 25^{\mathrm{i}}$ & $1.487(3)$ \\
\hline C6-H6A & 0.9800 & $\mathrm{C} 26-\mathrm{C} 27$ & $1.382(2)$ \\
\hline C6-H6B & 0.9800 & $\mathrm{C} 26-\mathrm{H} 26$ & 0.9500 \\
\hline $\mathrm{C} 6-\mathrm{H} 6 \mathrm{C}$ & 0.9800 & $\mathrm{C} 27-\mathrm{H} 27$ & 0.9500 \\
\hline $\mathrm{C} 8-\mathrm{C} 9$ & $1.5202(18)$ & & \\
\hline $\mathrm{N} 3-\mathrm{Cd}-\mathrm{S} 1$ & $121.64(3)$ & $\mathrm{O} 2-\mathrm{C} 9-\mathrm{C} 8$ & $107.32(10)$ \\
\hline $\mathrm{N} 3-\mathrm{Cd}-\mathrm{S} 3$ & $112.61(3)$ & $\mathrm{O} 2-\mathrm{C} 9-\mathrm{H} 9 \mathrm{~A}$ & 110.3 \\
\hline $\mathrm{S} 1-\mathrm{Cd}-\mathrm{S} 3$ & $125.725(11)$ & $\mathrm{C} 8-\mathrm{C} 9-\mathrm{H} 9 \mathrm{~A}$ & 110.3 \\
\hline $\mathrm{N} 3-\mathrm{Cd}-\mathrm{S} 2$ & $95.71(3)$ & $\mathrm{O} 2-\mathrm{C} 9-\mathrm{H} 9 \mathrm{~B}$ & 110.3 \\
\hline $\mathrm{S} 1-\mathrm{Cd}-\mathrm{S} 2$ & $69.571(10)$ & $\mathrm{C} 8-\mathrm{C} 9-\mathrm{H} 9 \mathrm{~B}$ & 110.3 \\
\hline $\mathrm{S} 3-\mathrm{Cd}-\mathrm{S} 2$ & $104.839(11)$ & $\mathrm{H} 9 \mathrm{~A}-\mathrm{C} 9-\mathrm{H} 9 \mathrm{~B}$ & 108.5 \\
\hline $\mathrm{N} 3-\mathrm{Cd}-\mathrm{S} 4$ & $98.43(3)$ & $\mathrm{N} 2-\mathrm{C} 10-\mathrm{C} 12$ & $112.15(11)$ \\
\hline $\mathrm{S} 1-\mathrm{Cd}-\mathrm{S} 4$ & $102.645(11)$ & $\mathrm{N} 2-\mathrm{C} 10-\mathrm{C} 11$ & $109.64(12)$ \\
\hline $\mathrm{S} 3-\mathrm{Cd}-\mathrm{S} 4$ & $69.533(10)$ & $\mathrm{C} 12-\mathrm{C} 10-\mathrm{C} 11$ & $112.07(13)$ \\
\hline $\mathrm{S} 2-\mathrm{Cd}-\mathrm{S} 4$ & $165.865(11)$ & $\mathrm{N} 2-\mathrm{C} 10-\mathrm{H} 10$ & 107.6 \\
\hline $\mathrm{C} 1-\mathrm{S} 1-\mathrm{Cd}$ & $87.22(4)$ & $\mathrm{C} 12-\mathrm{C} 10-\mathrm{H} 10$ & 107.6 \\
\hline $\mathrm{C} 1-\mathrm{S} 2-\mathrm{Cd}$ & $84.39(4)$ & $\mathrm{C} 11-\mathrm{C} 10-\mathrm{H} 10$ & 107.6 \\
\hline $\mathrm{C} 7-\mathrm{S} 3-\mathrm{Cd}$ & $87.13(4)$ & $\mathrm{C} 10-\mathrm{C} 11-\mathrm{H} 11 \mathrm{~A}$ & 109.5 \\
\hline $\mathrm{C} 7-\mathrm{S} 4-\mathrm{Cd}$ & $83.88(5)$ & $\mathrm{C} 10-\mathrm{C} 11-\mathrm{H} 11 \mathrm{~B}$ & 109.5 \\
\hline $\mathrm{C} 3-\mathrm{O} 1-\mathrm{H} 1 \mathrm{O}$ & $106.5(13)$ & $\mathrm{H} 11 \mathrm{~A}-\mathrm{C} 11-\mathrm{H} 11 \mathrm{~B}$ & 109.5 \\
\hline $\mathrm{C} 9-\mathrm{O} 2-\mathrm{H} 2 \mathrm{O}$ & $105.2(13)$ & $\mathrm{C} 10-\mathrm{C} 11-\mathrm{H} 11 \mathrm{C}$ & 109.5 \\
\hline $\mathrm{C} 1-\mathrm{N} 1-\mathrm{C} 2$ & $120.10(10)$ & $\mathrm{H} 11 \mathrm{~A}-\mathrm{C} 11-\mathrm{H} 11 \mathrm{C}$ & 109.5 \\
\hline $\mathrm{C} 1-\mathrm{N} 1-\mathrm{C} 4$ & $121.37(10)$ & $\mathrm{H} 11 \mathrm{~B}-\mathrm{C} 11-\mathrm{H} 11 \mathrm{C}$ & 109.5 \\
\hline $\mathrm{C} 2-\mathrm{N} 1-\mathrm{C} 4$ & $118.36(10)$ & $\mathrm{C} 10-\mathrm{C} 12-\mathrm{H} 12 \mathrm{~A}$ & 109.5 \\
\hline $\mathrm{C} 7-\mathrm{N} 2-\mathrm{C} 8$ & $120.56(11)$ & $\mathrm{C} 10-\mathrm{C} 12-\mathrm{H} 12 \mathrm{~B}$ & 109.5 \\
\hline $\mathrm{C} 7-\mathrm{N} 2-\mathrm{C} 10$ & $121.95(11)$ & $\mathrm{H} 12 \mathrm{~A}-\mathrm{C} 12-\mathrm{H} 12 \mathrm{~B}$ & 109.5 \\
\hline $\mathrm{C} 8-\mathrm{N} 2-\mathrm{C} 10$ & $117.13(11)$ & $\mathrm{C} 10-\mathrm{C} 12-\mathrm{H} 12 \mathrm{C}$ & 109.5 \\
\hline $\mathrm{C} 13-\mathrm{N} 3-\mathrm{C} 17$ & $117.88(11)$ & $\mathrm{H} 12 \mathrm{~A}-\mathrm{C} 12-\mathrm{H} 12 \mathrm{C}$ & 109.5 \\
\hline $\mathrm{C} 13-\mathrm{N} 3-\mathrm{Cd}$ & $122.21(8)$ & $\mathrm{H} 12 \mathrm{~B}-\mathrm{C} 12-\mathrm{H} 12 \mathrm{C}$ & 109.5 \\
\hline $\mathrm{C} 17-\mathrm{N} 3-\mathrm{Cd}$ & $119.83(8)$ & $\mathrm{N} 3-\mathrm{C} 13-\mathrm{C} 14$ & $122.62(12)$ \\
\hline $\mathrm{C} 20-\mathrm{N} 4-\mathrm{C} 21$ & $117.14(12)$ & $\mathrm{N} 3-\mathrm{C} 13-\mathrm{H} 13$ & 118.7 \\
\hline $\mathrm{C} 27-\mathrm{N} 5-\mathrm{C} 23$ & $115.51(13)$ & $\mathrm{C} 14-\mathrm{C} 13-\mathrm{H} 13$ & 118.7 \\
\hline $\mathrm{N} 1-\mathrm{C} 1-\mathrm{S} 2$ & $121.29(9)$ & $\mathrm{C} 13-\mathrm{C} 14-\mathrm{C} 15$ & $119.85(12)$ \\
\hline $\mathrm{N} 1-\mathrm{C} 1-\mathrm{S} 1$ & $120.01(9)$ & $\mathrm{C} 13-\mathrm{C} 14-\mathrm{H} 14$ & 120.1 \\
\hline $\mathrm{S} 2-\mathrm{C} 1-\mathrm{S} 1$ & $118.69(7)$ & $\mathrm{C} 15-\mathrm{C} 14-\mathrm{H} 14$ & 120.1 \\
\hline $\mathrm{N} 1-\mathrm{C} 2-\mathrm{C} 3$ & $111.34(10)$ & $\mathrm{C} 16-\mathrm{C} 15-\mathrm{C} 14$ & $117.23(11)$ \\
\hline $\mathrm{N} 1-\mathrm{C} 2-\mathrm{H} 2 \mathrm{~A}$ & 109.4 & $\mathrm{C} 16-\mathrm{C} 15-\mathrm{C} 18$ & $121.11(11)$ \\
\hline $\mathrm{C} 3-\mathrm{C} 2-\mathrm{H} 2 \mathrm{~A}$ & 109.4 & $\mathrm{C} 14-\mathrm{C} 15-\mathrm{C} 18$ & $121.64(11)$ \\
\hline $\mathrm{N} 1-\mathrm{C} 2-\mathrm{H} 2 \mathrm{~B}$ & 109.4 & $\mathrm{C} 17-\mathrm{C} 16-\mathrm{C} 15$ & $119.62(11)$ \\
\hline $\mathrm{C} 3-\mathrm{C} 2-\mathrm{H} 2 \mathrm{~B}$ & 109.4 & $\mathrm{C} 17-\mathrm{C} 16-\mathrm{H} 16$ & 120.2 \\
\hline $\mathrm{H} 2 \mathrm{~A}-\mathrm{C} 2-\mathrm{H} 2 \mathrm{~B}$ & 108.0 & $\mathrm{C} 15-\mathrm{C} 16-\mathrm{H} 16$ & 120.2 \\
\hline $\mathrm{O} 1-\mathrm{C} 3-\mathrm{C} 2$ & $109.17(10)$ & N3-C17-C16 & $122.77(12)$ \\
\hline $\mathrm{O} 1-\mathrm{C} 3-\mathrm{H} 3 \mathrm{~A}$ & 109.8 & $\mathrm{~N} 3-\mathrm{C} 17-\mathrm{H} 17$ & 118.6 \\
\hline $\mathrm{C} 2-\mathrm{C} 3-\mathrm{H} 3 \mathrm{~A}$ & 109.8 & $\mathrm{C} 16-\mathrm{C} 17-\mathrm{H} 17$ & 118.6 \\
\hline
\end{tabular}




\begin{tabular}{|c|c|c|c|}
\hline $\mathrm{O} 1-\mathrm{C} 3-\mathrm{H} 3 \mathrm{~B}$ & 109.8 & $\mathrm{C} 19-\mathrm{C} 18-\mathrm{C} 22$ & $117.47(12)$ \\
\hline $\mathrm{C} 2-\mathrm{C} 3-\mathrm{H} 3 \mathrm{~B}$ & 109.8 & $\mathrm{C} 19-\mathrm{C} 18-\mathrm{C} 15$ & $121.90(12)$ \\
\hline $\mathrm{H} 3 \mathrm{~A}-\mathrm{C} 3-\mathrm{H} 3 \mathrm{~B}$ & 108.3 & $\mathrm{C} 22-\mathrm{C} 18-\mathrm{C} 15$ & $120.62(11)$ \\
\hline $\mathrm{N} 1-\mathrm{C} 4-\mathrm{C} 5$ & $109.96(11)$ & $\mathrm{C} 20-\mathrm{C} 19-\mathrm{C} 18$ & $119.37(13)$ \\
\hline $\mathrm{N} 1-\mathrm{C} 4-\mathrm{C} 6$ & $112.47(11)$ & $\mathrm{C} 20-\mathrm{C} 19-\mathrm{H} 19$ & 120.3 \\
\hline $\mathrm{C} 5-\mathrm{C} 4-\mathrm{C} 6$ & $112.12(12)$ & $\mathrm{C} 18-\mathrm{C} 19-\mathrm{H} 19$ & 120.3 \\
\hline $\mathrm{N} 1-\mathrm{C} 4-\mathrm{H} 4$ & 107.3 & $\mathrm{~N} 4-\mathrm{C} 20-\mathrm{C} 19$ & $123.24(13)$ \\
\hline $\mathrm{C} 5-\mathrm{C} 4-\mathrm{H} 4$ & 107.3 & $\mathrm{~N} 4-\mathrm{C} 20-\mathrm{H} 20$ & 118.4 \\
\hline $\mathrm{C} 6-\mathrm{C} 4-\mathrm{H} 4$ & 107.3 & $\mathrm{C} 19-\mathrm{C} 20-\mathrm{H} 20$ & 118.4 \\
\hline $\mathrm{C} 4-\mathrm{C} 5-\mathrm{H} 5 \mathrm{~A}$ & 109.5 & $\mathrm{~N} 4-\mathrm{C} 21-\mathrm{C} 22$ & $123.81(13)$ \\
\hline $\mathrm{C} 4-\mathrm{C} 5-\mathrm{H} 5 \mathrm{~B}$ & 109.5 & $\mathrm{~N} 4-\mathrm{C} 21-\mathrm{H} 21$ & 118.1 \\
\hline $\mathrm{H} 5 \mathrm{~A}-\mathrm{C} 5-\mathrm{H} 5 \mathrm{~B}$ & 109.5 & $\mathrm{C} 22-\mathrm{C} 21-\mathrm{H} 21$ & 118.1 \\
\hline $\mathrm{C} 4-\mathrm{C} 5-\mathrm{H} 5 \mathrm{C}$ & 109.5 & $\mathrm{C} 21-\mathrm{C} 22-\mathrm{C} 18$ & $118.98(12)$ \\
\hline $\mathrm{H} 5 \mathrm{~A}-\mathrm{C} 5-\mathrm{H} 5 \mathrm{C}$ & 109.5 & $\mathrm{C} 21-\mathrm{C} 22-\mathrm{H} 22$ & 120.5 \\
\hline $\mathrm{H} 5 \mathrm{~B}-\mathrm{C} 5-\mathrm{H} 5 \mathrm{C}$ & 109.5 & $\mathrm{C} 18-\mathrm{C} 22-\mathrm{H} 22$ & 120.5 \\
\hline $\mathrm{C} 4-\mathrm{C} 6-\mathrm{H} 6 \mathrm{~A}$ & 109.5 & $\mathrm{~N} 5-\mathrm{C} 23-\mathrm{C} 24$ & $124.26(14)$ \\
\hline $\mathrm{C} 4-\mathrm{C} 6-\mathrm{H} 6 \mathrm{~B}$ & 109.5 & $\mathrm{~N} 5-\mathrm{C} 23-\mathrm{H} 23$ & 117.9 \\
\hline $\mathrm{H} 6 \mathrm{~A}-\mathrm{C} 6-\mathrm{H} 6 \mathrm{~B}$ & 109.5 & $\mathrm{C} 24-\mathrm{C} 23-\mathrm{H} 23$ & 117.9 \\
\hline $\mathrm{C} 4-\mathrm{C} 6-\mathrm{H} 6 \mathrm{C}$ & 109.5 & $\mathrm{C} 23-\mathrm{C} 24-\mathrm{C} 25$ & $119.73(14)$ \\
\hline $\mathrm{H} 6 \mathrm{~A}-\mathrm{C} 6-\mathrm{H} 6 \mathrm{C}$ & 109.5 & $\mathrm{C} 23-\mathrm{C} 24-\mathrm{H} 24$ & 120.1 \\
\hline $\mathrm{H} 6 \mathrm{~B}-\mathrm{C} 6-\mathrm{H} 6 \mathrm{C}$ & 109.5 & $\mathrm{C} 25-\mathrm{C} 24-\mathrm{H} 24$ & 120.1 \\
\hline $\mathrm{N} 2-\mathrm{C} 7-\mathrm{S} 4$ & $121.13(10)$ & $\mathrm{C} 26-\mathrm{C} 25-\mathrm{C} 24$ & $116.13(13)$ \\
\hline $\mathrm{N} 2-\mathrm{C} 7-\mathrm{S} 3$ & $119.58(10)$ & $\mathrm{C} 26-\mathrm{C} 25-\mathrm{C} 25^{\mathrm{i}}$ & $121.98(15)$ \\
\hline $\mathrm{S} 4-\mathrm{C} 7-\mathrm{S} 3$ & $119.30(8)$ & $\mathrm{C} 24-\mathrm{C} 25-\mathrm{C} 25^{\mathrm{i}}$ & $121.88(15)$ \\
\hline $\mathrm{N} 2-\mathrm{C} 8-\mathrm{C} 9$ & $111.65(10)$ & $\mathrm{C} 27-\mathrm{C} 26-\mathrm{C} 25$ & $119.81(13)$ \\
\hline $\mathrm{N} 2-\mathrm{C} 8-\mathrm{H} 8 \mathrm{~A}$ & 109.3 & $\mathrm{C} 27-\mathrm{C} 26-\mathrm{H} 26$ & 120.1 \\
\hline $\mathrm{C} 9-\mathrm{C} 8-\mathrm{H} 8 \mathrm{~A}$ & 109.3 & $\mathrm{C} 25-\mathrm{C} 26-\mathrm{H} 26$ & 120.1 \\
\hline $\mathrm{N} 2-\mathrm{C} 8-\mathrm{H} 8 \mathrm{~B}$ & 109.3 & $\mathrm{~N} 5-\mathrm{C} 27-\mathrm{C} 26$ & $124.56(14)$ \\
\hline $\mathrm{C} 9-\mathrm{C} 8-\mathrm{H} 8 \mathrm{~B}$ & 109.3 & N5-C27-H27 & 117.7 \\
\hline $\mathrm{H} 8 \mathrm{~A}-\mathrm{C} 8-\mathrm{H} 8 \mathrm{~B}$ & 108.0 & $\mathrm{C} 26-\mathrm{C} 27-\mathrm{H} 27$ & 117.7 \\
\hline $\mathrm{C} 2-\mathrm{N} 1-\mathrm{C} 1-\mathrm{S} 2$ & $-176.55(9)$ & $\mathrm{C} 17-\mathrm{N} 3-\mathrm{C} 13-\mathrm{C} 14$ & $1.25(19)$ \\
\hline $\mathrm{C} 4-\mathrm{N} 1-\mathrm{C} 1-\mathrm{S} 2$ & $-1.29(16)$ & $\mathrm{Cd}-\mathrm{N} 3-\mathrm{C} 13-\mathrm{C} 14$ & $-175.45(10)$ \\
\hline $\mathrm{C} 2-\mathrm{N} 1-\mathrm{C} 1-\mathrm{S} 1$ & $3.28(16)$ & $\mathrm{N} 3-\mathrm{C} 13-\mathrm{C} 14-\mathrm{C} 15$ & $-0.6(2)$ \\
\hline $\mathrm{C} 4-\mathrm{N} 1-\mathrm{C} 1-\mathrm{S} 1$ & $178.54(9)$ & $\mathrm{C} 13-\mathrm{C} 14-\mathrm{C} 15-\mathrm{C} 16$ & $-1.00(19)$ \\
\hline $\mathrm{Cd}-\mathrm{S} 2-\mathrm{C} 1-\mathrm{N} 1$ & $-176.80(10)$ & $\mathrm{C} 13-\mathrm{C} 14-\mathrm{C} 15-\mathrm{C} 18$ & $177.49(12)$ \\
\hline $\mathrm{Cd}-\mathrm{S} 2-\mathrm{C} 1-\mathrm{S} 1$ & $3.36(6)$ & $\mathrm{C} 14-\mathrm{C} 15-\mathrm{C} 16-\mathrm{C} 17$ & $1.82(18)$ \\
\hline $\mathrm{Cd}-\mathrm{S} 1-\mathrm{C} 1-\mathrm{N} 1$ & $176.69(10)$ & $\mathrm{C} 18-\mathrm{C} 15-\mathrm{C} 16-\mathrm{C} 17$ & $-176.68(12)$ \\
\hline $\mathrm{Cd}-\mathrm{S} 1-\mathrm{C} 1-\mathrm{S} 2$ & $-3.48(7)$ & $\mathrm{C} 13-\mathrm{N} 3-\mathrm{C} 17-\mathrm{C} 16$ & $-0.37(19)$ \\
\hline $\mathrm{C} 1-\mathrm{N} 1-\mathrm{C} 2-\mathrm{C} 3$ & $-83.28(14)$ & $\mathrm{Cd}-\mathrm{N} 3-\mathrm{C} 17-\mathrm{C} 16$ & $176.41(9)$ \\
\hline $\mathrm{C} 4-\mathrm{N} 1-\mathrm{C} 2-\mathrm{C} 3$ & $101.32(13)$ & $\mathrm{C} 15-\mathrm{C} 16-\mathrm{C} 17-\mathrm{N} 3$ & $-1.19(19)$ \\
\hline $\mathrm{N} 1-\mathrm{C} 2-\mathrm{C} 3-\mathrm{O} 1$ & $173.57(10)$ & $\mathrm{C} 16-\mathrm{C} 15-\mathrm{C} 18-\mathrm{C} 19$ & $-152.60(13)$ \\
\hline $\mathrm{C} 1-\mathrm{N} 1-\mathrm{C} 4-\mathrm{C} 5$ & $-93.77(14)$ & $\mathrm{C} 14-\mathrm{C} 15-\mathrm{C} 18-\mathrm{C} 19$ & $28.96(19)$ \\
\hline $\mathrm{C} 2-\mathrm{N} 1-\mathrm{C} 4-\mathrm{C} 5$ & $81.57(14)$ & $\mathrm{C} 16-\mathrm{C} 15-\mathrm{C} 18-\mathrm{C} 22$ & $28.66(18)$ \\
\hline $\mathrm{C} 1-\mathrm{N} 1-\mathrm{C} 4-\mathrm{C} 6$ & $140.52(12)$ & $\mathrm{C} 14-\mathrm{C} 15-\mathrm{C} 18-\mathrm{C} 22$ & $-149.77(13)$ \\
\hline $\mathrm{C} 2-\mathrm{N} 1-\mathrm{C} 4-\mathrm{C} 6$ & $-44.15(15)$ & $\mathrm{C} 22-\mathrm{C} 18-\mathrm{C} 19-\mathrm{C} 20$ & $0.4(2)$ \\
\hline $\mathrm{C} 8-\mathrm{N} 2-\mathrm{C} 7-\mathrm{S} 4$ & $-178.55(9)$ & $\mathrm{C} 15-\mathrm{C} 18-\mathrm{C} 19-\mathrm{C} 20$ & $-178.33(13)$ \\
\hline $\mathrm{C} 10-\mathrm{N} 2-\mathrm{C} 7-\mathrm{S} 4$ & $-5.59(17)$ & $\mathrm{C} 21-\mathrm{N} 4-\mathrm{C} 20-\mathrm{C} 19$ & $-0.3(2)$ \\
\hline
\end{tabular}




\begin{tabular}{llll}
$\mathrm{C} 8-\mathrm{N} 2-\mathrm{C} 7-\mathrm{S} 3$ & $1.70(16)$ & $\mathrm{C} 18-\mathrm{C} 19-\mathrm{C} 20-\mathrm{N} 4$ & $-0.1(2)$ \\
$\mathrm{C} 10-\mathrm{N} 2-\mathrm{C} 7-\mathrm{S} 3$ & $174.65(10)$ & $\mathrm{C} 20-\mathrm{N} 4-\mathrm{C} 21-\mathrm{C} 22$ & $0.3(2)$ \\
$\mathrm{Cd}-\mathrm{S} 4-\mathrm{C} 7-\mathrm{N} 2$ & $-176.04(10)$ & $\mathrm{N} 4-\mathrm{C} 21-\mathrm{C} 22-\mathrm{C} 18$ & $0.1(2)$ \\
$\mathrm{Cd}-\mathrm{S} 4-\mathrm{C} 7-\mathrm{S} 3$ & $3.71(7)$ & $\mathrm{C} 19-\mathrm{C} 18-\mathrm{C} 22-\mathrm{C} 21$ & $-0.45(19)$ \\
$\mathrm{Cd}-\mathrm{S} 3-\mathrm{C} 7-\mathrm{N} 2$ & $175.91(10)$ & $\mathrm{C} 15-\mathrm{C} 18-\mathrm{C} 22-\mathrm{C} 21$ & $178.35(12)$ \\
$\mathrm{Cd}-\mathrm{S} 3-\mathrm{C} 7-\mathrm{S} 4$ & $-3.85(7)$ & $\mathrm{C} 27-\mathrm{N} 5-\mathrm{C} 23-\mathrm{C} 24$ & $0.4(2)$ \\
$\mathrm{C} 7-\mathrm{N} 2-\mathrm{C} 8-\mathrm{C} 9$ & $-84.72(15)$ & $\mathrm{N} 5-\mathrm{C} 23-\mathrm{C} 24-\mathrm{C} 25$ & $-0.2(3)$ \\
$\mathrm{C} 10-\mathrm{N} 2-\mathrm{C} 8-\mathrm{C} 9$ & $102.00(13)$ & $\mathrm{C} 23-\mathrm{C} 24-\mathrm{C} 25-\mathrm{C} 26$ & $-0.1(2)$ \\
$\mathrm{N} 2-\mathrm{C} 8-\mathrm{C} 9-\mathrm{O} 2$ & $178.55(11)$ & $\mathrm{C} 23-\mathrm{C} 24-\mathrm{C} 25-\mathrm{C} 25^{\mathrm{i}}$ & $179.10(16)$ \\
$\mathrm{C} 7-\mathrm{N} 2-\mathrm{C} 10-\mathrm{C} 12$ & $138.65(13)$ & $\mathrm{C} 24-\mathrm{C} 25-\mathrm{C} 26-\mathrm{C} 27$ & $0.3(2)$ \\
$\mathrm{C} 8-\mathrm{N} 2-\mathrm{C} 10-\mathrm{C} 12$ & $-48.17(16)$ & $\mathrm{C} 25-\mathrm{C} 25-\mathrm{C} 26-\mathrm{C} 27$ & $-178.94(16)$ \\
$\mathrm{C} 7-\mathrm{N} 2-\mathrm{C} 10-\mathrm{C} 11$ & $-96.19(15)$ & $\mathrm{C} 23-\mathrm{N} 5-\mathrm{C} 27-\mathrm{C} 26$ & $-0.2(2)$ \\
$\mathrm{C} 8-\mathrm{N} 2-\mathrm{C} 10-\mathrm{C} 11$ & $76.99(15)$ & $\mathrm{C} 25-\mathrm{C} 26-\mathrm{C} 27-\mathrm{N} 5$ & $-0.1(2)$ \\
\hline
\end{tabular}

Symmetry code: (i) $-x,-y+2,-z$.

Hydrogen-bond geometry $\left(A,{ }^{\circ}\right)$

$\mathrm{Cg} 1$ is the centroid of the $\mathrm{Zn} / \mathrm{S} 3 / \mathrm{S} 4 / \mathrm{C} 7$ chelate ring.

\begin{tabular}{lllll}
\hline$D-\mathrm{H} \cdots A$ & $D-\mathrm{H}$ & $\mathrm{H} \cdots A$ & $D \cdots A$ & $D-\mathrm{H} \cdots A$ \\
\hline $\mathrm{O} 1-\mathrm{H} 1 O \cdots \mathrm{N} 4^{4 i}$ & $0.83(2)$ & $1.88(2)$ & $2.7085(15)$ & $176(1)$ \\
$\mathrm{O} 2-\mathrm{H} 2 O \cdots \mathrm{O} 1^{\mathrm{iii}}$ & $0.83(1)$ & $1.89(1)$ & $2.7162(14)$ & $173(2)$ \\
$\mathrm{C} 4-\mathrm{H} 4 \cdots \mathrm{S} 2^{\mathrm{iv}}$ & 1.00 & 2.68 & $3.5395(14)$ & 144 \\
$\mathrm{C} 22-\mathrm{H} 22 \cdots \mathrm{O} 2^{\mathrm{v}}$ & 0.95 & 2.40 & $3.3473(17)$ & 174 \\
$\mathrm{C} 26-\mathrm{H} 26 \cdots C g 1^{\mathrm{vi}}$ & 0.95 & 3.00 & $3.776(3)$ & 140 \\
\hline
\end{tabular}

Symmetry codes: (ii) $-x, y-1,-z+1 / 2$; (iii) $x+1 / 2, y+1 / 2, z$; (iv) $x+1 / 2, y+3 / 2, z$; (v) $x,-y, z-1 / 2$; (vi) $x, y+1, z$. 